\title{
MIMO Antenna Polynomial Weighted Average Design Method of Downward-Looking Array SAR
}

\author{
Hui Liu, Qing Xu, Guowang Jin, and Hongmin Zhang \\ Institute of Geospatial Information, Information Engineering University, Zhengzhou 450052, China \\ Correspondence should be addressed to Qing Xu; xq@szdcec.com
}

Received 8 November 2016; Revised 25 January 2017; Accepted 6 February 2017; Published 12 April 2017

Academic Editor: Wen-Qin Wang

Copyright (C) 2017 Hui Liu et al. This is an open access article distributed under the Creative Commons Attribution License, which permits unrestricted use, distribution, and reproduction in any medium, provided the original work is properly cited.

\begin{abstract}
MIMO antenna polynomial weighted average design method of downward-looking array SAR was proposed from the angle of surveying and mapping in this paper, in order to solve the ill-posed problem that an equivalent virtual array can be implemented by a variety of physical transmitter-receiver arrays for bistatic MIMO linear array. For wave band, resolution, elevation precision, and working height concerned by the applications of surveying and mapping, the length of equivalent virtual array and actual physical array meeting the needs of large scale topographical mapping was solved. Then array numbers and position vectors of MIMO downward-looking array SAR of real aerial mapping platform were optimized. According to this design, some simulation experiments and comparisons were processed. The results proved the rationality and effectiveness of this array configuration by comparing the differences of 3D imaging results and the original simulation scene, counting mean and standard deviation of elevation reconstruction error eliminating the influence of shadow areas, and counting the probability of elevation reconstruction error within half a resolution of the whole scene and individual building area.
\end{abstract}

\section{Introduction}

As a new type of three-dimensional synthetic aperture radar (3D-SAR) system, downward-looking array SAR has been paid more and more attention by scholars both at home and abroad. In this system, a linear array antenna is distributed along the cross-track direction, working at downwardlooking model. And the three-dimensional resolution in azimuth direction, range direction, and cross-track direction is, respectively, achieved by synthetic aperture technique, pulse compression technique, and beamforming technique $[1,2]$. The new system effectively resolves the problems about geometric distortion, the blind of the bottom, and the left and right fuzzy of the traditional SAR system. It plays an important role in three-dimensional reconstruction of urban areas, topographic survey of complex terrain, disaster prediction and assessment, aircraft navigation, and blind landing.

The cross-track resolution of downward-looking array SAR depends on the length of the array antenna. The longer the length of the array is, the higher the resolution of this dimension will be. Then, as the number of required array elements increases more, the design cost and the implementation complexity of the linear array antenna will double, which will make it impossible for us to physically implement such a large array. The introduction of multiple input multiple output (MIMO) technology [3] solves this problem. If we want to obtain the equivalent array with $n$ virtual array elements, we can reduce the number of real array elements to $2 \sqrt{n}$ by reasonable arrangement of the array elements' positions, thus greatly reducing the hardware cost and system complexity.

At present, MIMO radar array design is mainly realized by the principle of spatial convolution $[4,5]$ and equivalent phase center (EPC) [6]. In 2006, the airborne radar for threedimensional imaging and nadir observation (ARTINO) system was developed by the FGAN-FHR, which used the EPC principle to form 1440 virtual array elements with a spacing of $0.0028 \mathrm{~m}$ [7-11]. In 2010, through the derivation of the spatial convolution principle, Su et al. proposed the method using self-convolutional inverse operation to solve the position of transmitter-receiver common array element [12]. Mitra et al. introduced the array pattern and performed variable substitution and factorization of its analytic expression, so 
as to realize the design of uniform superposition equivalent array [13]. Chen et al. realized the design of broadband MIMO radar array by combining two uniform linear arrays into an equivalent uniform array [14]. Du et al. proposed an optimized array antenna disposal way for MIMO downwardlooking 3D-SAR based on the EPC principle [1]. Zhang et al. proposed a novel MIMO radar array structure optimization algorithm with the highest array element utilization [15]. In 2011, Wang Q. studied the method of MIMO radar array design using polynomial theory [16]. Ye et al. optimized the antenna configuration of a "one-active" sparse array 3D-SAR imaging system with two-terminal-transmission and midreception mode by the EPC principle [17]. In 2012, Peng et al. constructed array antenna configuration of MIMO downward-looking airborne 3D-SAR [18]. Li et al. proposed an improved ARTINO antenna structure, compact midtransmission model, and compact mid-reception model [19]. In 2013, Wang et al. designed the MIMO antenna array with the polynomial factorization method [20]. Wu et al. solved the multigroup MIMO linear array design scheme by the spatial convolution inverse operation and analyzed the advantages and disadvantages of each group's design scheme, which provided technical support for the actual construction of airborne linear array 3D-SAR imaging system [21]. In 2014, Yu et al. proposed a MIMO-SAR array configuration method with mid-transmission and two-terminal-reception mode and proved that this model can get better imaging performance in downward-looking 3D imaging [22]. Li et al. gave a sparse array optimization design method based on simulated annealing algorithm [23]. However, for the array configuration of aerial photogrammetric aircraft platform commonly used in surveying and mapping, there is no specific research in the existing literatures.

It is an ill-posed problem to obtain the actual physical transmitter-receiver array by a given equivalent virtual array for MIMO linear array [21]. If the number of the transmitting array elements and the number of the receiving array elements are factors of the number of equivalent array elements without 1 and itself, the same equivalent virtual array can be constructed by exchange multiplication, which brings the difficulties and opportunities for the optimal design of the array. In this paper, MIMO antenna polynomial weighted average design method of downward-looking array SAR is introduced from the angle of surveying and mapping. Wave band, resolution, elevation precision, and working height concerned by surveying and mapping application are introduced; the array number and position vectors of MIMO downward-looking array SAR of real mapping aerial platform are optimized. According to this configuration, some simulation experiments are carried out, proving that the array configuration is rationality and effectiveness.

\section{Relationship between Convolution and Polynomial Weighted Average}

An example of polynomial multiplication is firstly introduced:

$$
\begin{aligned}
(x+1)\left(x^{2}+3 x+2\right)= & \left(x^{3}+3 x^{2}+2 x\right) \\
& +\left(x^{2}+3 x+2\right) \\
= & x^{3}+4 x^{2}+5 x+2 .
\end{aligned}
$$

As shown in Figure 1, the coefficients of polynomial $x+1$ are recorded as $[a(1), a(0)]=[1,1]$, which are arranged in descending order. The coefficients of polynomial $x^{2}+3 x+$ 2 are recorded as $[b(2), b(1), b(0)]=[1,3,2]$, which are arranged in ascending order. Firstly, let the highest power of the two polynomials be vertically aligned and multiply. Then whenever the ascending polynomial shifts one term to the right, the vertically aligned terms of the two polynomials are multiplied one time, until the lowest power of the two polynomials is aligned. Finally, the result of the multiplication is added to obtain the result polynomial, just as $[c(3), c(2), c(1), c(0)]=[1,4,5,2]$, which is the coefficients of polynomial $x^{3}+4 x^{2}+5 x+2$.

Through the calculation method in Figure 1, it is easy to get

$$
\begin{aligned}
& c(3)=a(0) b(3)+a(1) b(2)+a(2) b(1) \\
& +a(3) b(0) \\
& c(2)=a(0) b(2)+a(1) b(1)+a(2) b(0), \\
& c(1)=a(0) b(1)+a(1) b(0), \\
& c(0)=a(0) b(0) \text {. }
\end{aligned}
$$

Without loss of generality, we suppose the polynomial coefficients are $a(n), n=0 \sim s$ and $b(n), n=0 \sim t$, and the result polynomial coefficients can be obtained by multiplying the two polynomials:

$$
c(n)=\sum_{k=0}^{n} a(k) b(n-k), \quad n=0 \sim(s+t) .
$$

It is not difficult to find that (3) is also a convolution expression for $a(n)$ and $b(n)$. Therefore, we associate the convolution with the polynomial weighted average. We can take $c(n)$ as the weighted average for function $b(n)$ to function $a(n)$, where $b(n)$ is for the weight role and $a(n)$ is for the averaged role.

Literatures $[4,5,12]$ have pointed out that the positional relationship of the equivalent virtual array is determined by the spatial position convolution of the transmitting array element and receiving array element under certain conditions. Therefore, from the above analysis, we can see that the equivalent virtual array positional relationship can also be obtained by polynomial weighted average of the spatial position of the transmitting array element and receiving array element under certain conditions.

\section{Design Principle of Polynomial Weighted Average for Cross-Track MIMO Antenna Array}

The imaging geometry relationship of the MIMO downwardlooking array SAR system is shown in Figure 2 (4 transmitting antennas and 4 receiving antennas). The sparse array antennas distributed along the wing are divided into two parts: the transmitting antenna subarray $T_{y}$ and the receiving 

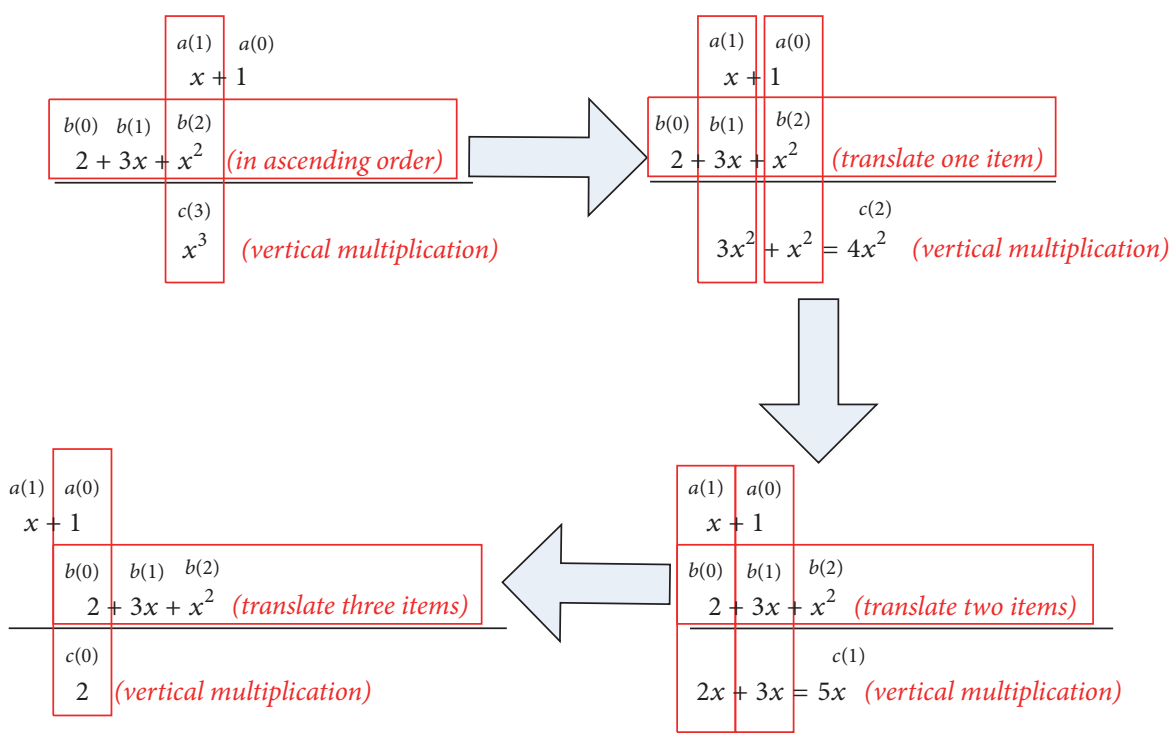

FIGURE 1: Relationship between convolution and polynomial weighted average.

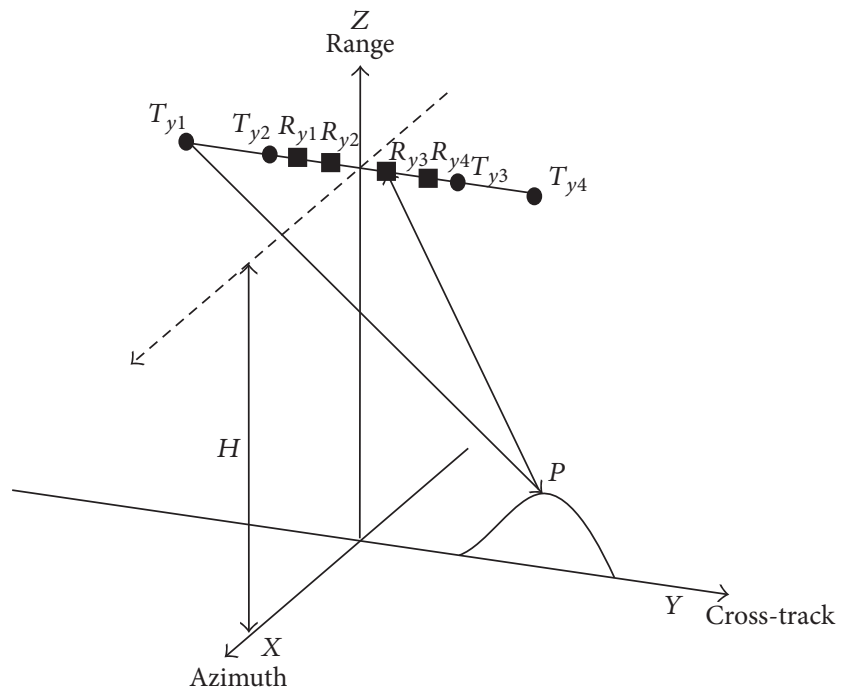

Transmitting array element

Receiving array element

FIGURE 2: The imaging geometry relationship of the MIMO downward-looking array SAR system.

antenna subarray $R_{y}$, in which $T_{y}$ contains $M$ transmitting array elements and $R_{y}$ contains $N$ receiving array elements.

From the above analysis, we can see that both the transmitting array and the receiving array can be represented by a polynomial. The position of each element has a certain relation with the power of the polynomial. As shown in (4), we suppose that an array corresponds to a unique polynomial $y(x)$ :

$$
y(x)=\sum_{n=0}^{K} w(n) x^{n}
$$

where $n$ is a power corresponding to the position of array elements, $K$ is the number of array elements, and $w(n)$ is the coefficient of the $n$th term. For a physical array, $w(n)$ marks whether there are array elements at the position indicated by the power, just as (5). For a virtual array, it marks the overlapping times of the virtual array element at the position indicated by the power, which represents the weight of the virtual array element:

$$
w(n)= \begin{cases}1, & \text { there exists array element } \\
0, & \text { there does not exists array element. }\end{cases}
$$

MIMO antenna design of array SAR is the process of solving transmitting array polynomial and receiving array polynomial by virtual array polynomial. However, a polynomial may not be decomposed into products of two polynomials whose coefficients are positive integers. Therefore, the solution is not unique; that is to say, the transmitting array and the receiving array are not unique. So it needs to add certain constraint conditions to obtain the optimal solution to meet the requirements.

The conventional MIMO antenna design of array SAR used to adopt the two-terminal-transmission and midreception mode. That is, the transmitting antennas are evenly distributed at both ends of the whole array, and the receiving antennas are evenly distributed in between. However, the length of the actual physical array corresponding to this distribution is always longer than the length of the equivalent virtual array, which is intolerable to very limited cross-track spatial resources. Therefore, according to the symmetrical distribution characteristics of array SAR, this paper seeks an array distribution of equivalent virtual array and real physical array with the same length.

In order to avoid the problem that the nonuniform virtual array can degrade the spatial resolution and image quality of array SAR system, it is desirable that the coefficients of 
the virtual array polynomial are all 1, and the power of the independent variables is continuous. We suppose that the required uniform virtual array is polynomial $y_{K}(x)$ :

$$
y_{K}(x)=\sum_{n=0}^{K-1} x^{n}
$$

where $K$ is the number of virtual array elements and $K$ is even due to the symmetrical distribution characteristics. If $K$ can be expressed as the product of $M+1$ prime numbers,

$$
K=\prod_{m=0}^{M}(K)_{m}
$$

Then $y_{K}(x)$ can be expressed as $[16,20]$

$$
y_{K}(x)=\prod_{m=0}^{M} y_{(K)_{m}}\left(x^{S_{m}}\right),
$$

with

$$
S_{m}=\prod_{i=0}^{m}(K)_{i-1},
$$

where $(K)_{i-1}=1$.

Through combination multiplication of $M+1 y_{(K)_{m}}\left(x^{S_{m}}\right)$, we can get multiple sets of transmitting arrays and receiving arrays. However, it should be noted that the changes of the order of $(K)_{m}$ will affect the value of $S_{m}$.

MIMO antenna design of array SAR requires transmitting antennas to be distributed evenly at both ends, so the spatial position convolution of MIMO antenna array can be equivalent as follows: when the number of transmitting antennas is even, half of them can be placed at the other end of the array. Figure 3 shows an equivalent receiving array based on the principle of spatial position convolution, where 1,2 , and 3 represent the number of times that the virtual array element is overlapped at this position. Figure 4 shows the results when half of the transmitting antennas are placed at the opposite end of the array. The equivalent receiving array is exactly the same to the former.

Therefore, (8) should be added to some constraint conditions to obtain the antenna configuration formula of array SAR:

(1) Since the array elements need to be distributed symmetrically, the first prime number of the prime factorization of the equivalent virtual array must be 2 .

(2) Polynomial, whose all powers are even numbers, is the receiving array, and the power is the position of the receiving array element.

(3) Polynomial with uninterrupted arrangement power from 0 to $n-1$ is the transmitting array.

(4) According to the symmetrical distribution of the array, transmitting array polynomial is arranged in ascending order and the number of array elements is divided into two parts. The first $n / 2$ array elements are located at one end of the physical array, and the power is the position of the transmitting antenna. The left $n / 2$ array elements are located at the other end of the physical array, and (power $-n+k$ ) is the position of the transmitting antenna.

So (8) can be changed to

$$
y_{K}(x)=\prod_{m=0}^{M} y_{(K)_{m}}\left(x^{S_{m}}\right)
$$

s.t. $(K)_{0}=2$, and $K$ is an even number,

polynomial $\left(x^{0}+x^{2}+\cdots+x^{2^{n-1}}\right)$ is the receiving array, $n$ is a positive integer,

polynomial $\left(x^{0}+x^{1}+\cdots+x^{n-1}\right)$ is the transmitting array, $n$ is a positive integer,

the first $\frac{n}{2}$ array elements are located at one end of the physical array,

and the power is the position of the transmitting antenna,

the left $\frac{n}{2}$ array elements are located at the other end of the physical array,

and the (power $-n+k)$ is the position of the transmitting antenna.

As an example, we suppose $K=16$ and substitute it into (7). Under the premise of meeting the first constraint condition, 16 can be decomposed into 4 prime factors $16=$ $(K)_{0} \times(K)_{1} \times(K)_{2} \times(K)_{3}=2 \times 2 \times 2 \times 2$. Therefore, we can get 

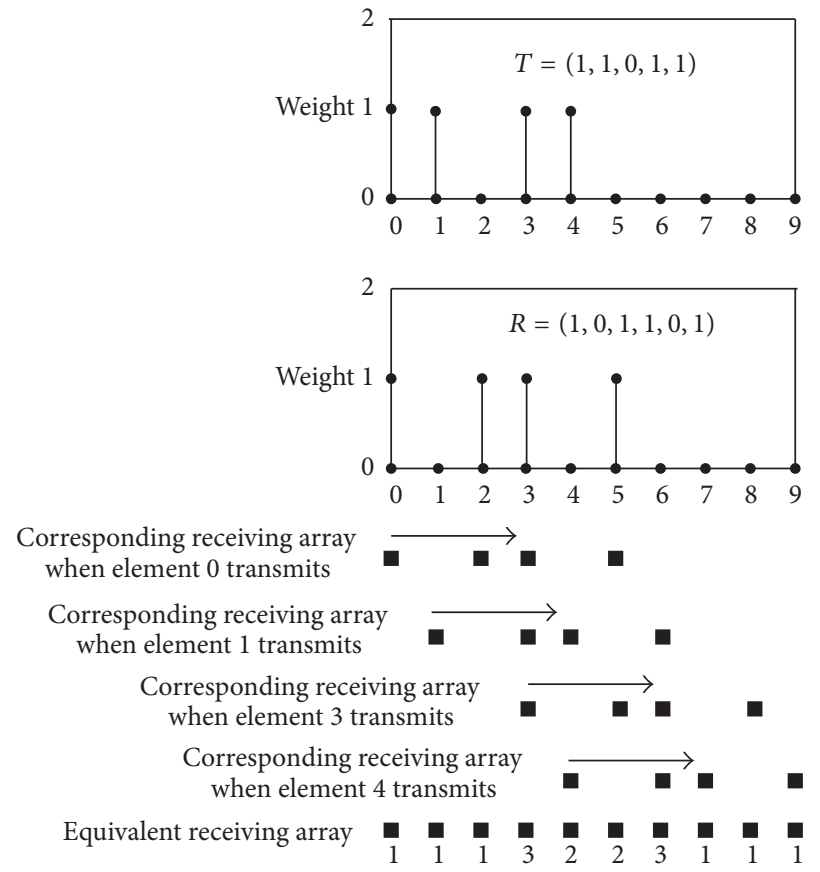

FIgURE 3: Spatial convolution of MIMO array.
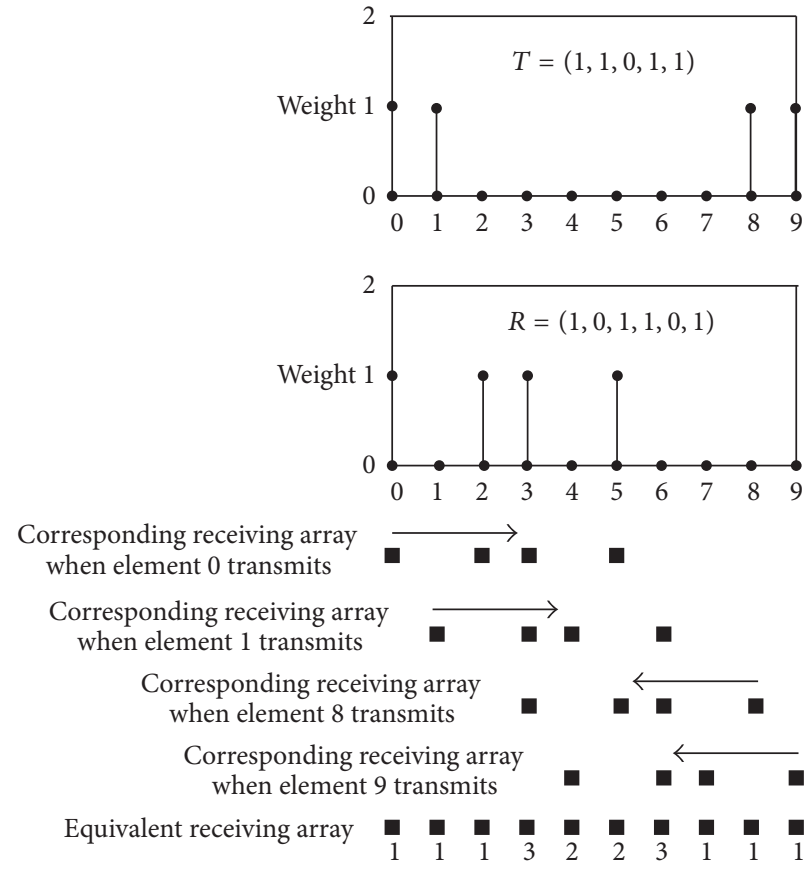

FIgURE 4: Equivalent array for spatial convolution.

$M=3$, substitute it into (11), and further obtain the virtual array polynomial.

$$
\begin{gathered}
y_{K}(x)=\prod_{m=0}^{3} y_{(K)_{m}}\left(x^{S_{m}}\right)=y_{(K)_{0}}\left(x^{S_{0}}\right) \cdot y_{(K)_{1}}\left(x^{S_{1}}\right) \\
\cdot y_{(K)_{2}}\left(x^{S_{2}}\right) \cdot y_{(K)_{3}}\left(x^{S_{3}}\right)=y_{(K)_{0}}\left(x^{(K)_{-1}}\right)
\end{gathered}
$$

$$
\begin{aligned}
& \cdot y_{(K)_{1}}\left(x^{(K)_{-1} \times(K)_{0}}\right) y_{(K)_{2}}\left(x^{(K)_{-1} \times(K)_{0} \times(K)_{1}}\right) \\
& \cdot y_{(K)_{3}}\left(x^{(K)_{-1} \times(K)_{0} \times(K)_{1} \times(K)_{2}}\right)=y_{2}\left(x^{1}\right) y_{2}\left(x^{1 \times 2}\right) \\
& \cdot y_{2}\left(x^{1 \times 2 \times 2}\right) y_{2}\left(x^{1 \times 2 \times 2 \times 2}\right)=\left(x^{0}+x^{1}\right) \\
& \cdot\left(\left(x^{2}\right)^{0}+\left(x^{2}\right)^{1}\right)\left(\left(x^{4}\right)^{0}+\left(x^{4}\right)^{1}\right)
\end{aligned}
$$




$$
\begin{aligned}
& \cdot\left(\left(x^{8}\right)^{0}+\left(x^{8}\right)^{1}\right)=(1+x)\left(1+x^{2}\right)\left(1+x^{4}\right) \\
& \cdot\left(1+x^{8}\right) .
\end{aligned}
$$

Combined with the constraint conditions in (10), we can get the transmitting array polynomial and receiving array polynomial meeting the requirements. The original twelve transmitter-receiver arrays available through any combination of factors have only left the following three:

\section{Combination 1}

$$
\begin{aligned}
& y_{T}(x)=1+x \text { averaged factor } \\
& y_{R}(x)=\left(1+x^{2}\right)\left(1+x^{4}\right)\left(1+x^{8}\right) \quad \text { weight, }
\end{aligned}
$$

\section{Combination 2}

$$
\begin{aligned}
& y_{T}(x)=(1+x)\left(1+x^{2}\right) \quad \text { averaged factor } \\
& y_{R}(x)=\left(1+x^{4}\right)\left(1+x^{8}\right) \quad \text { weight, }
\end{aligned}
$$

\section{Combination 3}

$$
\begin{aligned}
& y_{T}(x)=(1+x)\left(1+x^{2}\right)\left(1+x^{4}\right) \quad \text { averaged factor } \\
& y_{R}(x)=\left(1+x^{8}\right) \quad \text { weight. }
\end{aligned}
$$

Combination 1 . We consider the polynomial $1+x$ as averaged factor and the polynomial $\left(1+x^{2}\right)\left(1+x^{4}\right)\left(1+x^{8}\right)$ as weight. The polynomial $1+x$ with uninterrupted arrangement power is the transmitting array, and the polynomial $1+x^{2}+x^{4}+x^{6}+x^{8}+$ $x^{10}+x^{12}+x^{14}$ is the receiving array, whose all powers are even numbers after expansion. This combination corresponds to the actual physical array with 2 transmitting antennas and 8 receiving antennas. As shown in Figure 5, the positions of the transmitting array and receiving array are, respectively, $\{0,15\}$ and $\{0,2,4,6,8,10,12,14\}$.

Combination 2 . We consider the polynomial $(1+x)\left(1+x^{2}\right)$ as averaged factor and the polynomial $\left(1+x^{4}\right)\left(1+x^{8}\right)$ as weight. The polynomial $1+x+x^{2}+x^{3}$ with uninterrupted arrangement power is the transmitting array, and the polynomial $1+x^{4}+$ $x^{8}+x^{12}$ is the receiving array, whose all powers are even numbers after expansion. This combination corresponds to the actual physical array with 4 transmitting antennas and 4 receiving antennas. As shown in Figure 6, the positions of the transmitting array and receiving array are, respectively, $\{0,1,14,15\}$ and $\{0,4,8,12\}$.

Combination 3 . We consider the polynomial $(1+x)\left(1+x^{2}\right)(1+$ $\left.x^{4}\right)$ as averaged factor and the polynomial $\left(1+x^{8}\right)$ as weight. The polynomial $1+x+x^{2}+x^{3}+x^{4}+x^{5}+x^{6}+x^{7}+x^{8}$ with uninterrupted arrangement power is the transmitting array, and the polynomial $1+x^{8}$ is the receiving array, whose all powers are even numbers after expansion. This combination corresponds to the actual physical array with 8 transmitting antennas and 2 receiving antennas. As shown in Figure 7, the positions of the transmitting array and receiving array are, respectively, $\{0,1,2,3,12,13,14,15\}$ and $\{0,8\}$.

The length of virtual array obtained by EPC principle is always shorter than the length of the physical array, but the physical array designed by this method is equal in length to the virtual array. Therefore, in the case of limited crosstrack resources, this method has more advantages than EPC principle.

\section{MIMO Antenna Design of Downward-Looking Array SAR}

MIMO antenna design is the key to the physical realization of downward-looking array SAR airborne system. As shown in Figure 8, it is necessary to consider the problems about topographic mapping requirements, aircraft platform loads, radar device dimensions, cross-track sampling intervals (the spatial distribution of equivalent virtual elements should satisfy the spatial sampling law), and so on:

(1) Firstly, it should select the high-frequency band with more application potential and determine the interval of the equivalent virtual array element based on the sampling law (in order to avoid the influence of the grating lobe, the distance between two adjacent array elements should be less than or equal to half wavelength [24]).

(2) Secondly, according to the requirements about flight height and resolution of topographic mapping and the determined wavelength, the length of equivalent virtual array in corresponding scale mapping is solved.

(3) Then, the total number of the array elements is obtained by dividing the length of equivalent virtual array by the interval of equivalent virtual array element. The number of the transmitting arrays and receiving arrays is determined by analyzing the decomposable factors of total number.

(4) Finally, the position of the array element in the actual airborne platform is determined by the corresponding method.

In this paper, MIMO antenna array configuration of the real mapping aerial platform is studied in an alternate transmitting mode. In which, only one transmitting array element transmits signal for each pulse repetition time, and all receiving array elements receive the echo of the scene synchronously. After all transmitting array elements sequentially transmit signal, a uniform virtual array with equal spacing can be obtained.

4.1. Topographic Accuracy Requirements. As shown in Table 1, the following requirements for topographic accuracy are listed in the specification named "Basic Geographic Information 3D Model Product Specification" [25]. In this paper, we study MIMO antenna configuration of downward-looking array SAR based on above specification. In which, the index of restraining the cross-track resolution of array SAR is "grid 

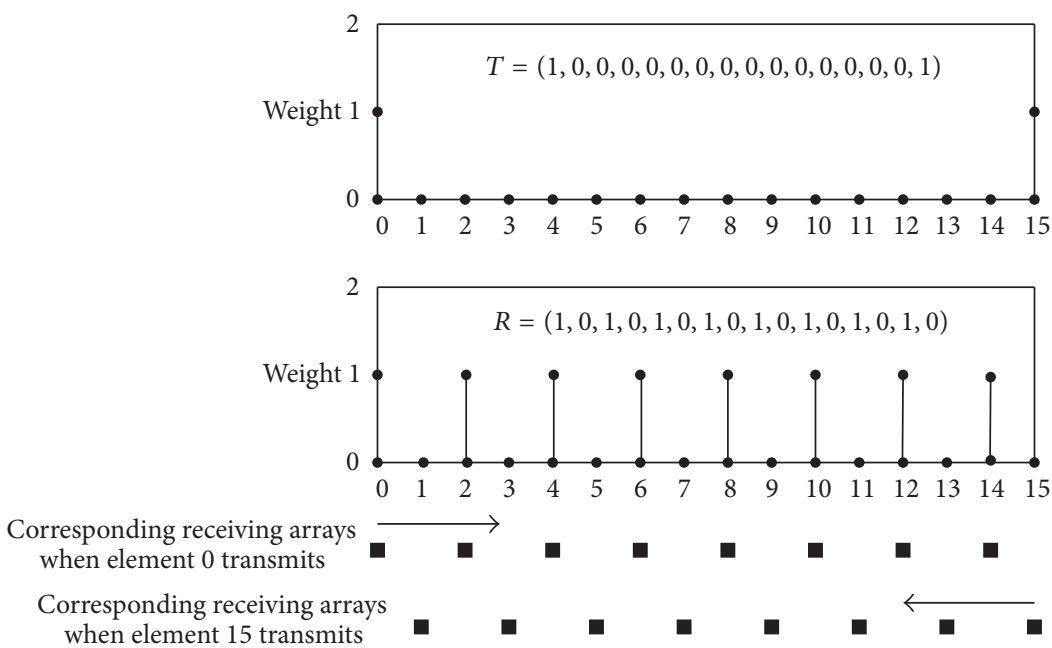

Equivalent receiving array

FIGURE 5: "2-transmitter 8-receiver" transmitter-receiver array and the equivalent receiving array.
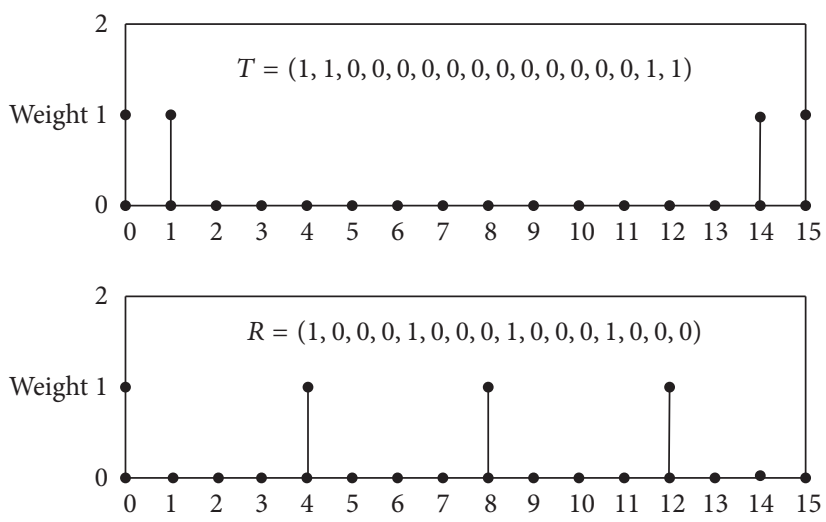

Corresponding receiving arrays when element 0 transmits

Corresponding receiving arrays when element 1 transmits

Corresponding receiving arrays when element 14 transmits

Corresponding receiving arrays when element 15 transmits

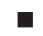

-
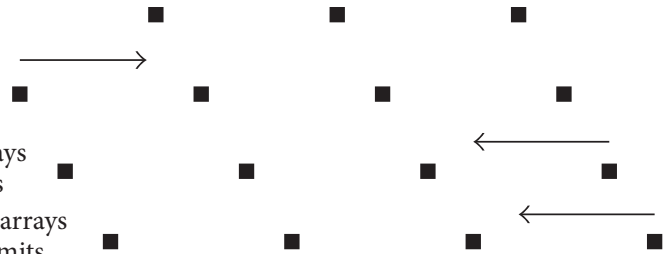

Equivalent receiving array

FIGURE 6: “4-transmitter 4-receiver” transmitter-receiver array and the equivalent receiving array.

TABLE 1: 3D topographic accuracy.

\begin{tabular}{lccccc}
\hline Mapping scale & Grid spacing/m & MSE of flat ground $/ \mathrm{m}$ & MSE of hills/m & MSE of mountains/m & MSE of high mountains $/ \mathrm{m}$ \\
\hline $1: 500$ & 0.5 & 0.37 & 0.75 & 1.05 & 1.5 \\
$1: 1000$ & 1 & 0.37 & 1.05 & 1.5 & 3 \\
$1: 2000$ & 2 & 0.75 & 1.05 & 2.25 & 3 \\
\hline
\end{tabular}



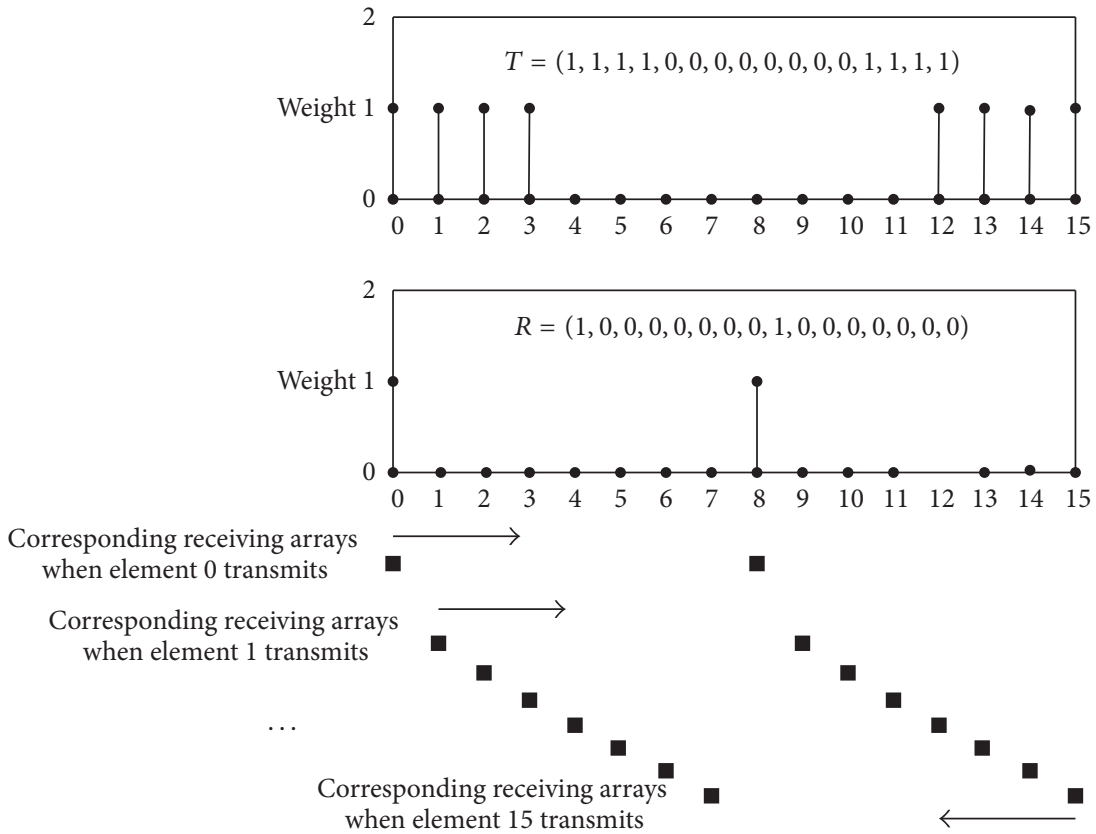

$\square$

Equivalent receiving array

FIGURE 7: "8-transmitter 2-receiver" transmitter-receiver array and the equivalent receiving array.

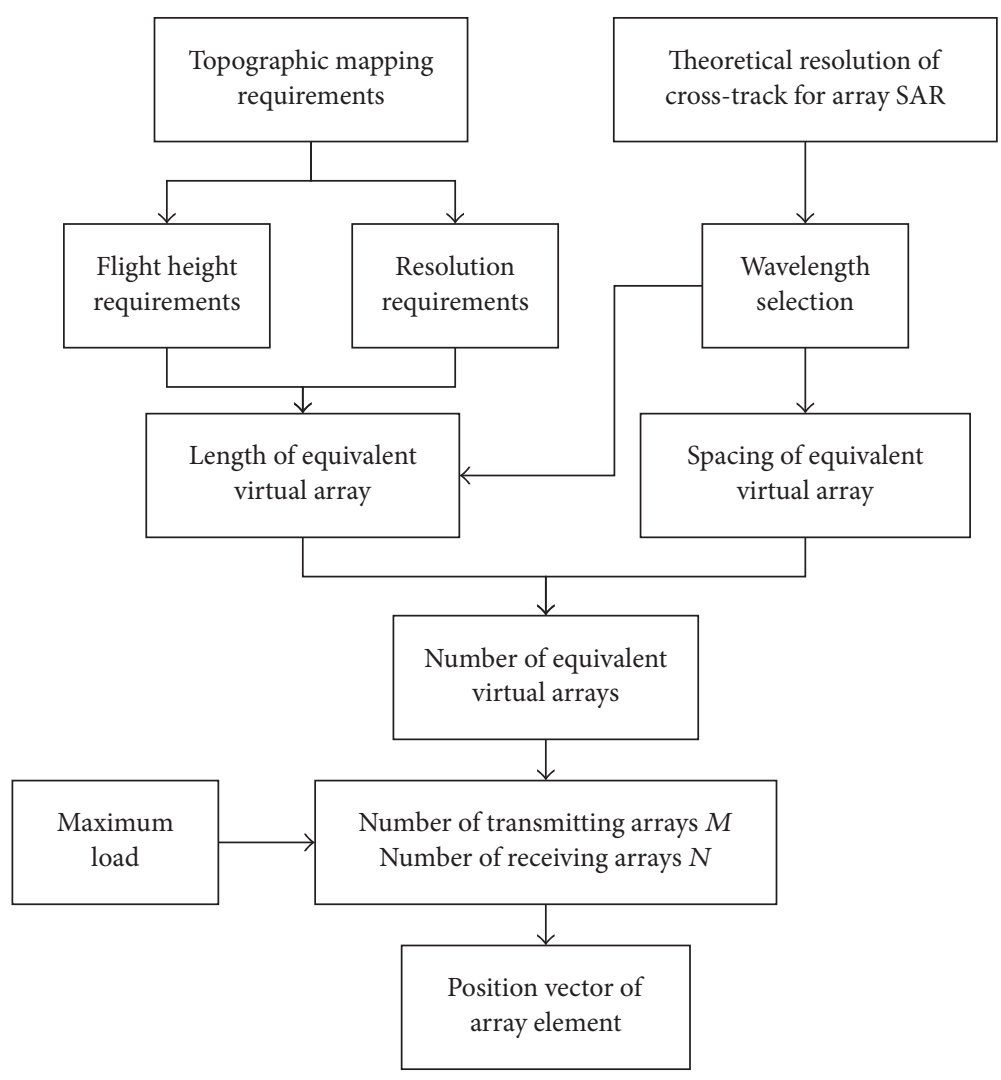

Figure 8: Flow chart of array antenna design. 
TABLE 2: Parameters of common aerial photogrammetric aircraft.

\begin{tabular}{lcc}
\hline Aircraft & Wingspan/m & Maximum load $/ \mathrm{kg}$ \\
\hline Transport 5 & 14.236 & 1500 \\
Transport 12 & 17.235 & 1700 \\
PC-6 & 15.87 & 1130 \\
Air king C90GT & 15.32 & 877 \\
\hline
\end{tabular}

spacing," and the mean square error (MSE) of height in the last four columns restrains the range resolution of array SAR.

4.2. Cross-Track Resolution of MIMO Downward-Looking Array SAR. Literature [1] (p. 32) pointed out that the crosstrack resolution $\rho_{y}$ of the MIMO array SAR is

$$
\rho_{y}=\frac{\lambda H}{2 L}
$$

where $\lambda$ is the wavelength, $H$ is the platform flight height, and $L$ is the synthetic aperture length of the virtual array. Cross-track resolution is proportional to the wavelength and the flight height and inversely proportional to the synthetic aperture length. In order to obtain higher cross-track resolution, $\lambda$ and $H$ should be minimized, and $L$ should be larger. Therefore, the Ka-band that could obtain wide bandwidth and narrow beam and the low-altitude platform can be used to achieve the physical realization. Because this technology is limited to low-altitude mapping, it is not suitable for small and medium scale mapping but suitable for large scale mapping such as $1: 500,1: 1000$, and $1: 2000$.

4.3. Transmitter-Receiver Antenna Configuration. Airborne platform of array SAR generally includes two types: large aircraft and unmanned aerial vehicles. This paper only studies the former. In the surveying and mapping production, common aerial photogrammetric aircrafts include transport 5, transport 12, PC-6, and the air king, whose aircraft wingspan and maximum load are shown in Table 2. The wingspan directly limits the maximum length of the equivalent virtual array, and the maximum load determines the maximum number of array elements that the aircraft can tolerate.

Learning from the experience of optical aerial photography, flight height depends on the pixel size, focal length, and resolution. For different cameras, flight height is roughly distributed in the $580-8000 \mathrm{~m}$ range. When the mapping scales are, respectively, $1: 500,1: 1000$, and $1: 2000$, relative flying heights are generally around $600 \mathrm{~m}, 1200 \mathrm{~m}$, and $2500 \mathrm{~m}$. Thus, according to these height constraints of aerial photography, relative flying height of the corresponding scale can be used as a reference to analyzing the length of equivalent virtual array. Furthermore, the "Low-Altitude Digital Aerial Photography Specification" [26] also gives other height constraint conditions. The relative flying height of lowaltitude platform is generally not more than $1500 \mathrm{~m}$, and the maximum does not exceed $2000 \mathrm{~m}$. So the reference flying height of $1: 500,1: 1000$, and $1: 2000$ and the corresponding
3D topographic accuracy in Table 1 can be, respectively, substituted into (15):

$$
\begin{aligned}
& 1: 500 \quad 0.5=\frac{8 \times 10^{-3} \times 600}{2 L} \longrightarrow L_{\text {virtual }}=4.8 \mathrm{~m}, \\
& 1: 1000 \quad 1=\frac{8 \times 10^{-3} \times 1200}{2 L} \longrightarrow L_{\text {virtual }}=4.8 \mathrm{~m}, \\
& 1: 20002=\frac{8 \times 10^{-3} \times 2000}{2 L} \longrightarrow L_{\text {virtual }}=4 \mathrm{~m} .
\end{aligned}
$$

Because MIMO antenna design of downward-looking array SAR should be applicable to all large scale mapping tasks, we should take the maximum length of virtual array $L_{\text {virtual }}=4.8 \mathrm{~m}$. Combined with the array element spacing $S=\lambda / 2=4 \times 10^{-3} \mathrm{~m}$, the number of required array elements is $M_{\text {total }}=4.8 \mathrm{~m} / 4 \times 10^{-3} \mathrm{~m}+1=1201$. However, 1201 is a prime number and can not effectively represent the idea of array configuration. 1200, its approximate value, is used to discuss the polynomial weighted average design method. As shown in Table 3, 1200 equivalent virtual array elements include the following 28 kinds of transmitter-receiver combinations. In order to minimize the number of array elements and the length of actual array and reduce the complexity of industrial manufacturing, it is recommended to select the transmitter-receiver array with 30 transmitting antennas and 40 receiving antennas.

For the array with 1200 equivalent virtual array elements, 1200 can be decomposed into 7 prime factors, when $M$ is equal to 6:

$$
\begin{aligned}
1200= & (K)_{0} \times(K)_{1} \times(K)_{2} \times(K)_{3} \times(K)_{4} \times(K)_{5} \\
& \times(K)_{6}=2 \times 3 \times 5 \times 2 \times 2 \times 2 \times 5 .
\end{aligned}
$$

Substituting $M=6$ into (10), we can get the virtual array polynomial:

$$
\begin{aligned}
& y_{K}(x)=\prod_{m=0}^{6} y_{(K)_{m}}\left(x^{S_{m}}\right)=y_{(K)_{0}}\left(x^{S_{0}}\right) \cdot y_{(K)_{1}}\left(x^{S_{1}}\right) \\
& \cdot y_{(K)_{2}}\left(x^{S_{2}}\right) \cdot y_{(K)_{3}}\left(x^{S_{3}}\right) \cdot y_{(K)_{4}}\left(x^{S_{4}}\right) \cdot y_{(K)_{5}}\left(x^{S_{5}}\right) \\
& \cdot y_{(K)_{6}}\left(x^{S_{6}}\right)=y_{(K)_{0}}\left(x^{(K)_{-1}}\right) y_{(K)_{1}}\left(x^{(K)_{-1} \times(K)_{0}}\right) \\
& \cdot y_{(K)_{2}}\left(x^{(K)_{-1} \times(K)_{0} \times(K)_{1}}\right) y_{(K)_{3}}\left(x^{(K)_{-1} \times(K)_{0} \times(K)_{1} \times(K)_{2}}\right) \\
& \cdot y_{(K)_{4}}\left(x^{(K)_{-1} \times(K)_{0} \times(K)_{1} \times(K)_{2} \times(K)_{3}}\right) \\
& \cdot y_{(K)_{5}}\left(x^{(K)_{-1} \times(K)_{0} \times(K)_{1} \times(K)_{2} \times(K)_{3} \times(K)_{4}}\right) \\
& \cdot y_{(K)_{6}}\left(x^{(K)_{-1} \times(K)_{0} \times(K)_{1} \times(K)_{2} \times(K)_{3} \times(K)_{4} \times(K)_{5}}\right)
\end{aligned}
$$


TABLE 3: Transmitter-receiver combination.

\begin{tabular}{lllllll}
\hline $2 \times 600$ & $3 \times 400$ & $4 \times 300$ & $5 \times 240$ & $6 \times 200$ & $8 \times 150$ & $10 \times 120$ \\
$600 \times 2$ & $400 \times 3$ & $300 \times 4$ & $240 \times 5$ & $200 \times 6$ & $150 \times 8$ & $25 \times 48$ \\
$12 \times 100$ & $15 \times 80$ & $16 \times 75$ & $20 \times 60$ & $24 \times 50$ & $30 \times 40$ \\
$100 \times 12$ & $80 \times 15$ & $75 \times 16$ & $60 \times 20$ & $50 \times 24$ & $48 \times 25$ \\
\hline
\end{tabular}

TABLE 4: Simulation parameters of carrier aircraft system.

\begin{tabular}{|c|c|c|c|c|c|c|c|}
\hline $\begin{array}{l}\text { Number of equivalent } \\
\text { elements }\end{array}$ & PRF & Carrier frequency & $\begin{array}{l}\text { Sampling } \\
\text { frequency }\end{array}$ & Platform speed & Pulse width & Platform height & Bandwidth \\
\hline 1200 & $200 \mathrm{~Hz}$ & $37.5 \mathrm{GHZ}$ & $180 \mathrm{MHZ}$ & $100 \mathrm{~m} / \mathrm{s}$ & $0.1 \mu \mathrm{s}$ & $600 \mathrm{~m}$ & $150 \mathrm{MHZ}$ \\
\hline
\end{tabular}

$$
\begin{aligned}
& =y_{2}\left(x^{1}\right) y_{3}\left(x^{1 \times 2}\right) y_{5}\left(x^{1 \times 2 \times 3}\right) y_{2}\left(x^{1 \times 2 \times 3 \times 5}\right) \\
& \cdot y_{2}\left(x^{1 \times 2 \times 3 \times 5 \times 2}\right) y_{2}\left(x^{1 \times 2 \times 3 \times 5 \times 2 \times 2}\right) \\
& \cdot y_{5}\left(x^{1 \times 2 \times 3 \times 5 \times 2 \times 2 \times 2}\right)=\left(x^{0}+x^{1}\right)\left(\left(x^{2}\right)^{0}+\left(x^{2}\right)^{1}\right. \\
& \left.+\left(x^{2}\right)^{2}\right)\left(\left(x^{6}\right)^{0}+\left(x^{6}\right)^{1}+\left(x^{6}\right)^{2}+\left(x^{6}\right)^{3}+\left(x^{6}\right)^{4}\right) \\
& \cdot\left(\left(x^{30}\right)^{0}+\left(x^{30}\right)^{1}\right)\left(\left(x^{60}\right)^{0}+\left(x^{60}\right)^{1}\right)\left(\left(x^{120}\right)^{0}\right. \\
& \left.+\left(x^{120}\right)^{1}\right)\left(\left(x^{240}\right)^{0}+\left(x^{240}\right)^{1}+\left(x^{240}\right)^{2}+\left(x^{240}\right)^{3}\right. \\
& \left.+\left(x^{240}\right)^{4}\right)=\left[(1+x)\left(1+x^{2}+x^{4}\right)\right. \\
& \left.\cdot\left(1+x^{6}+x^{12}+x^{18}+x^{24}\right)\right]\left[\left(1+x^{30}\right)\left(1+x^{60}\right)\right. \\
& \left.\cdot\left(1+x^{120}\right)\left(1+x^{240}+x^{480}+x^{720}+x^{960}\right)\right]=(1 \\
& \left.+x+x^{2}+\cdots+x^{28}+x^{29}\right)\left(1+x^{30}+x^{60}+\cdots\right. \\
& \left.+x^{1140}+x^{1170}\right) .
\end{aligned}
$$

According to (10), the transmitting array of physical transmitter-receiver array with 30 transmitting antennas and 40 receiving antennas is

$\{0,1,2,3,4,5,6,7,8,9,10,11,12,13,14,1185,1186,1187$,

$1188,1189,1190,1191,1192,1193,1194,1195,1196$,

$1197,1198,1199\}$.

The receiving array is

$\{0,30,60,90,120,150,180,210,240,270,300,330,360$,

$390,420,450,480,510,540,570,600,630,660,690$,

$720,750,780,810,840,870,900,930,960,990,1020$,

$1050,1080,1110,1140,1170\}$.

\section{Experiment and Analysis}

In this paper, the MIMO antenna array configuration with 30 transmitting antennas and 40 receiving antennas is used to simulate the echo data of downward-looking 3D building scene, which is equivalent to 1200 virtual array elements. The simulation parameters are shown in Table 4. The original simulation scene is shown in Figure 9. There exists an L-type building with 30 meters height in the range of $200 \mathrm{~m} * 200 \mathrm{~m}$. According to the geometric model of downward-looking array SAR, the height transition between the building and the ground will inevitably produce shadow areas. The existence of shadows is determined by the radar incident angle calculated from the $3 \mathrm{D}$ position of the simulation building, and the backscattering coefficient of the shadow areas is set to zero. Figures 10 and 11, respectively, show the top and shadow information of the simulated building.

The original echo data is a chirp signal along range direction as shown in Figure 12. After three-dimensional (3D) range-Doppler (R-D) imaging processing, Figures 13, 14, and 15 give the section views of the azimuth-angle plane, the angle-range plane, and the azimuth-range plane, respectively. But these imaging results and the original terrain are not in the same coordinate system, so they can not be comparatively analyzed. The imaging results need to be interpolated with the same grid spacing as the original terrain according to the relationship of angle, slope range and height, and slant range. After being mapped in the same coordinate system, the simulation results can be obtained as shown in Figure 16. The original appearance of the L-type building is basically restored with only some glitches existing in the marginal area, which proves the effectiveness of the method. After the final imaging results are differentiated from the original terrain, the differential contrast image can be obtained. As shown in Figure 17, the red part of the figure shows that the original scene is higher than the reconstruction scene, and the blue part shows the original scene is lower than the reconstruction scene. In this figure, the edge area of the building is mostly red or blue, and the interior of the building is basically green, which indicates that the reconstruction height is more accurate in the interior of the building and the difference is larger in the edge area of the building. The reason is that low cross-track resolution, inaccurate shaded area heights, and inaccurate phase compensation will directly affect the imaging accuracy.

In order to further verify the effectiveness of the array design, we compare the imaging results of the method in this paper with the results obtained by spatial convolution principle and EPC principle. In order to ensure that the 


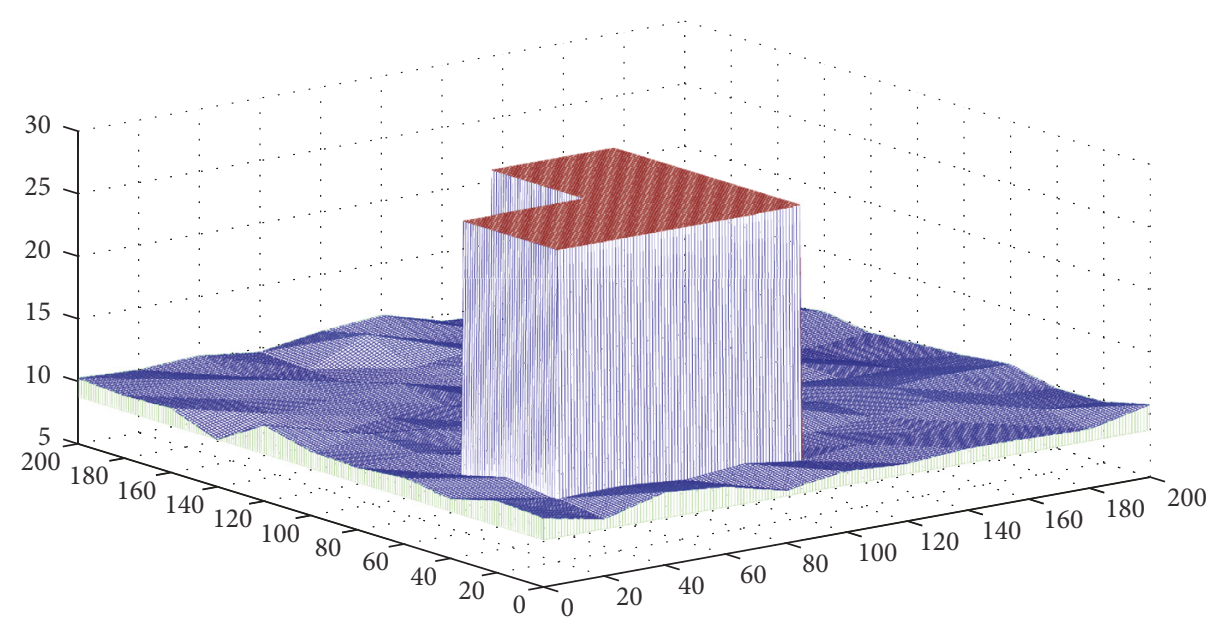

FIGURE 9: Original terrain.

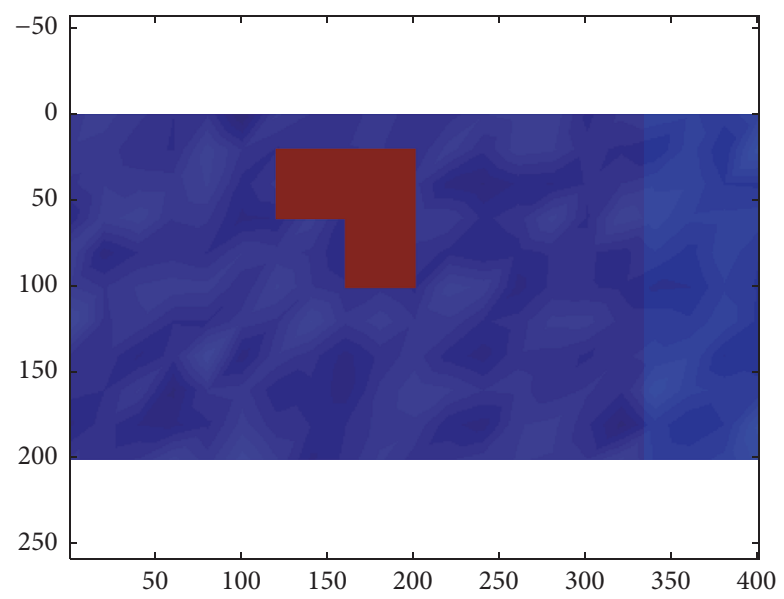

FIgURE 10: Top information of building.

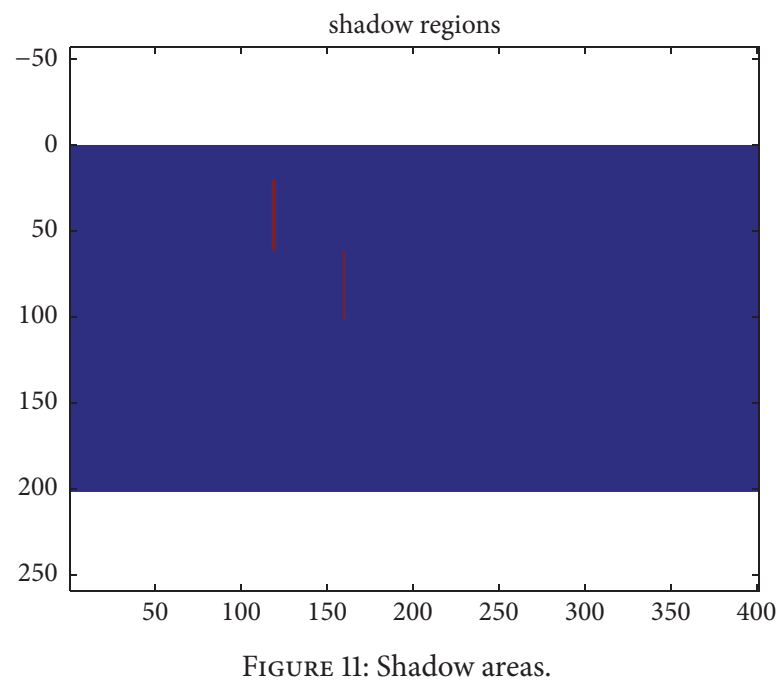




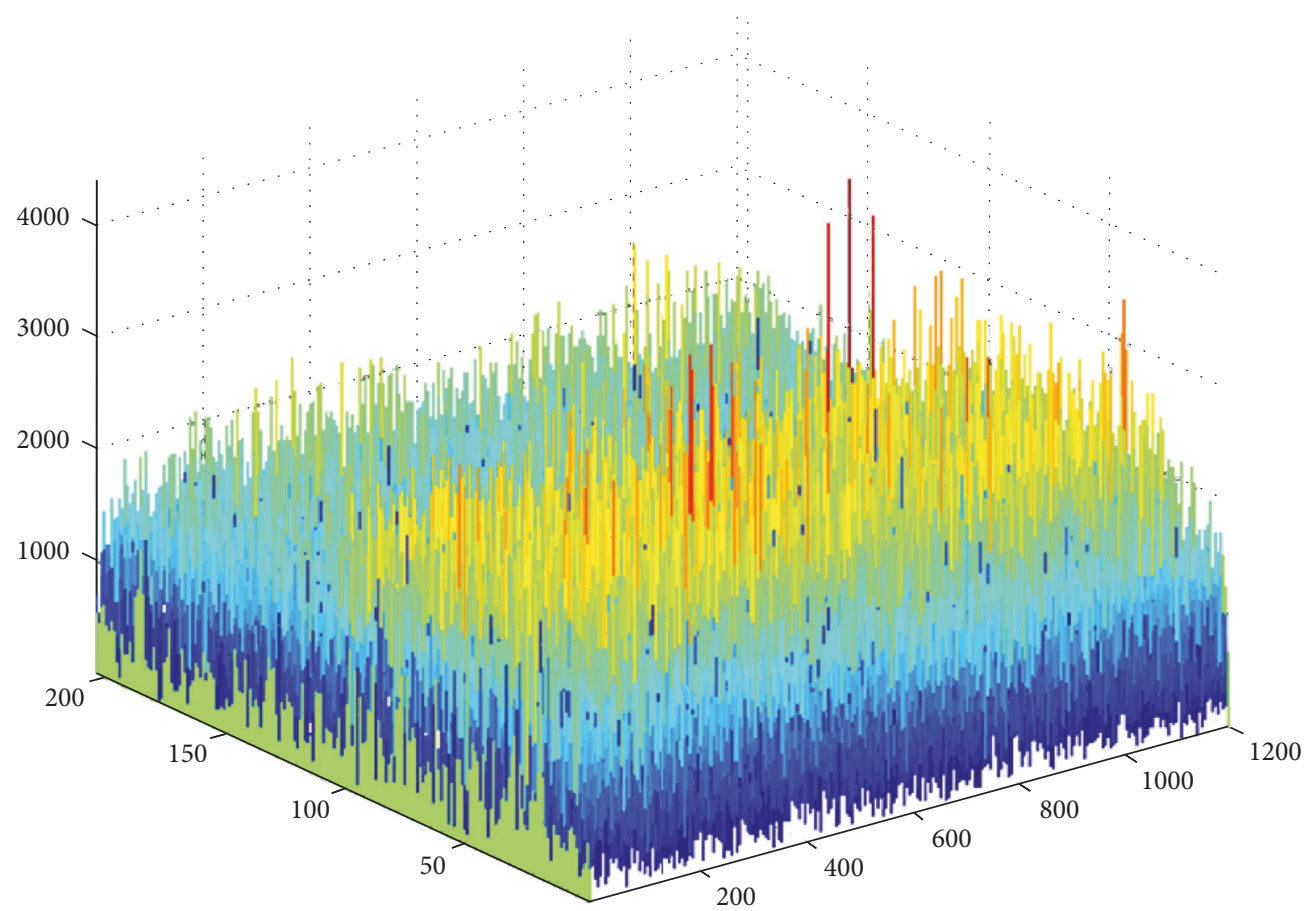

FIgURE 12: Original echo.
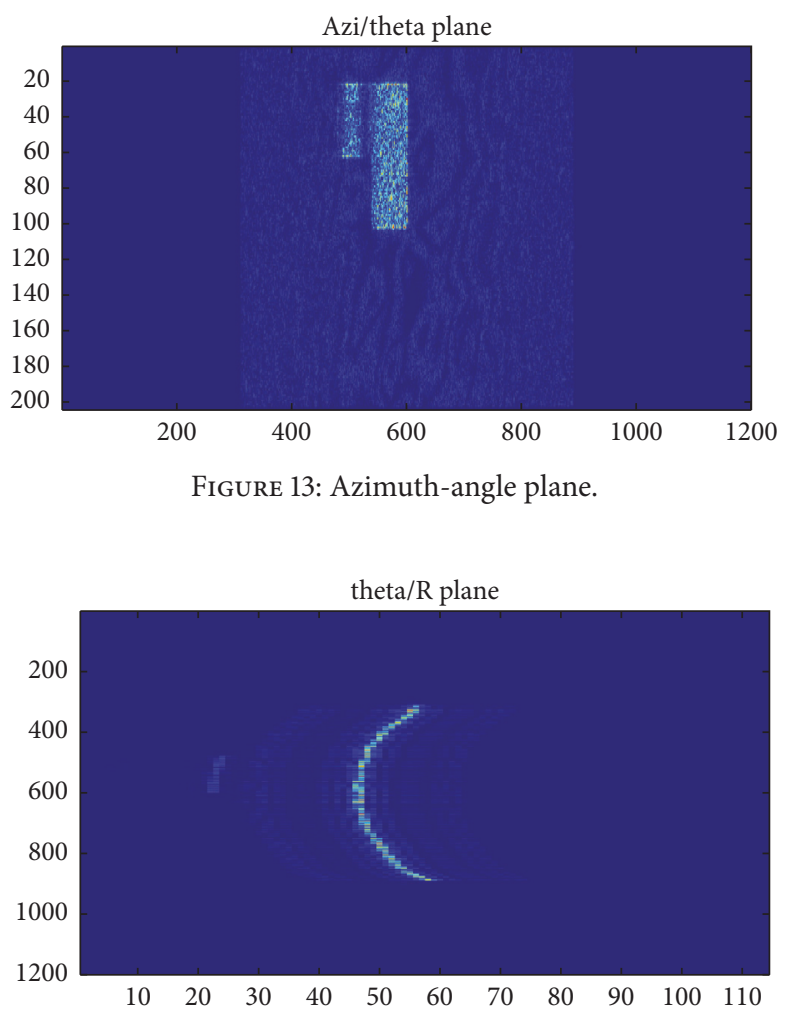

Figure 14: Angle-range plane. 


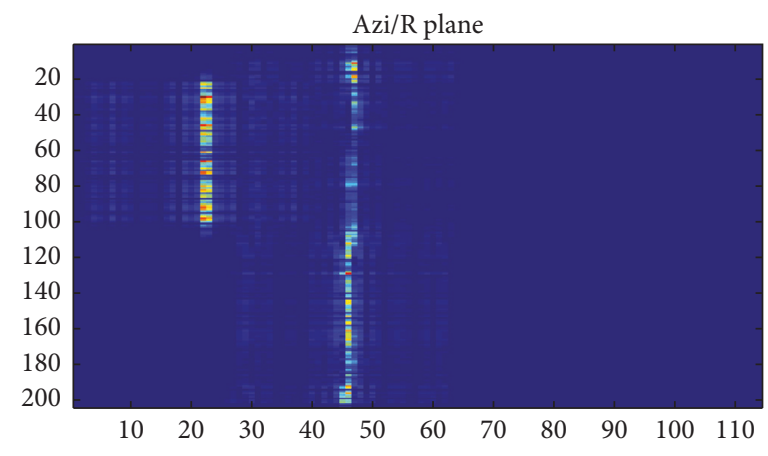

FIGURE 15: Azimuth-range plane.

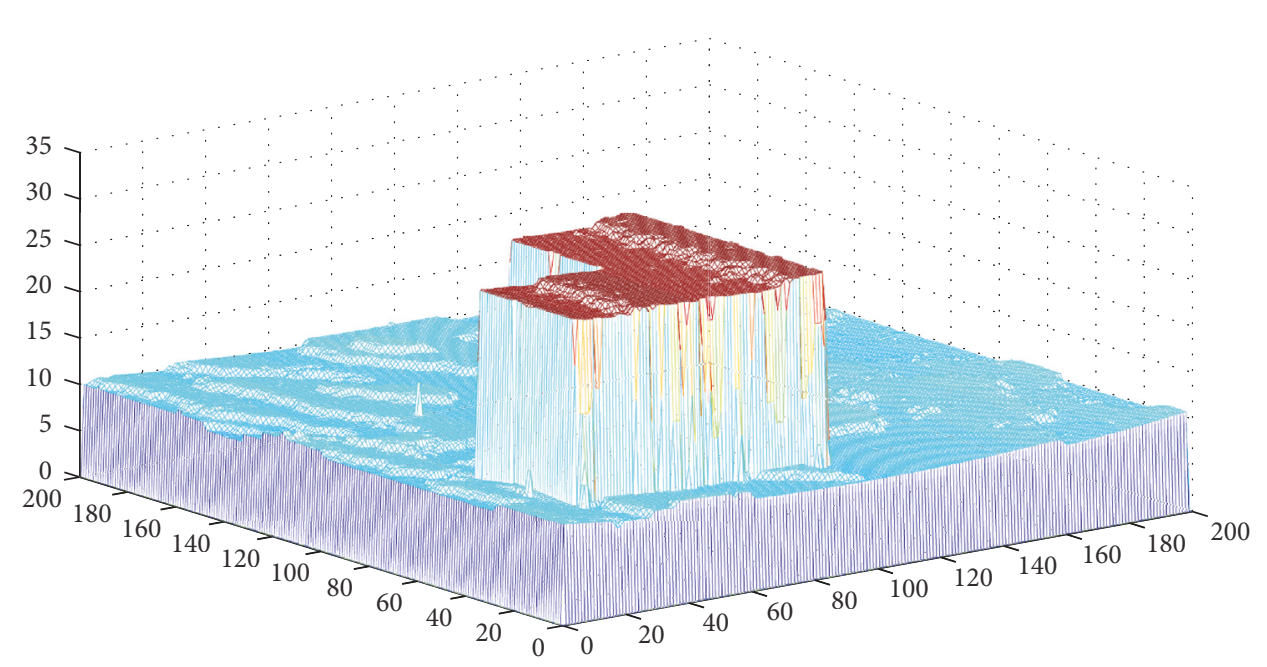

FIGURE 16: Imaging results.

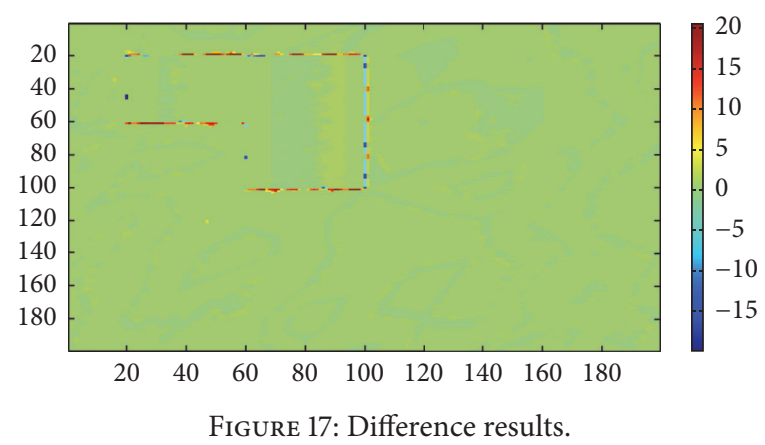

contrast reference is consistent, all three methods should adopt the array configuration with 30 transmitting antennas and 40 receiving antennas, which can be equivalent to 1200 virtual array elements. Different principles can lead to different distances between the transmitting and receiving array elements relative to the same target area, so the compensation phase is also different. In order to simplify the difficulty of imaging, image processing of each principle will do the appropriate approximation.

The equivalent virtual array obtained by spatial convolution principle is equal in length to the actual physical array. And the transmitting array is
$\{0,1,2,3,4,5,6,7,8,9,10,11,12,13,14,15,16,17,18,19,20$

$$
21,22,23,24,25,26,27,28,29\} \text {. }
$$

The receiving array is

$\{0,30,60,90,120,150,180,210,240,270,300,330,360$, $390,420,450,480,510,540,570,600,630,660,690$,

$720,750,780,810,840,870,900,930,960,990,1020$,

$1050,1080,1110,1140,1170\}$.

The imaging results corresponding to this proposed method are shown in Figures 18-23. 


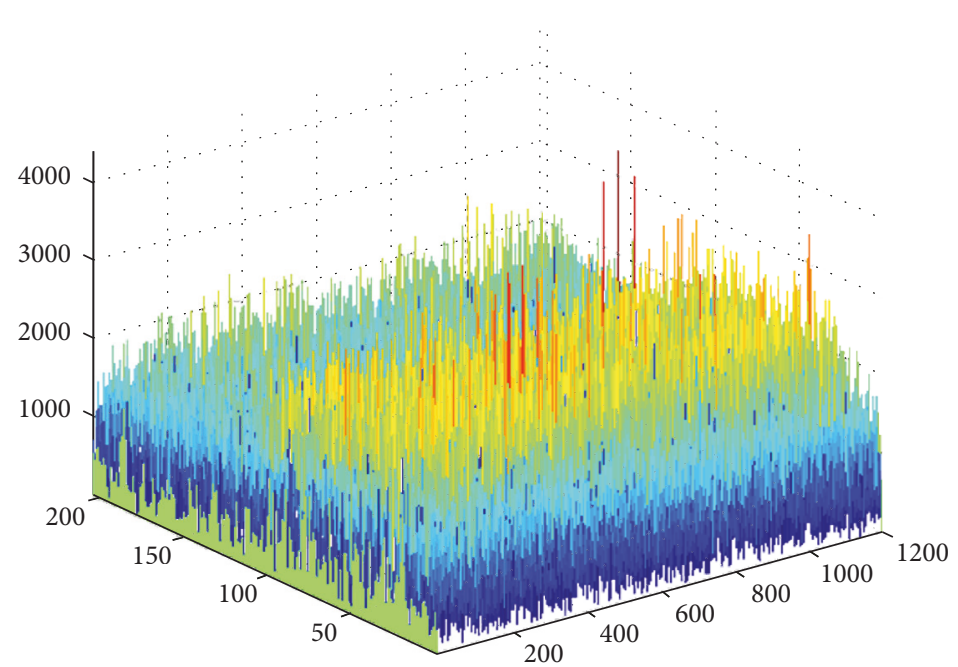

Figure 18: Original echo.

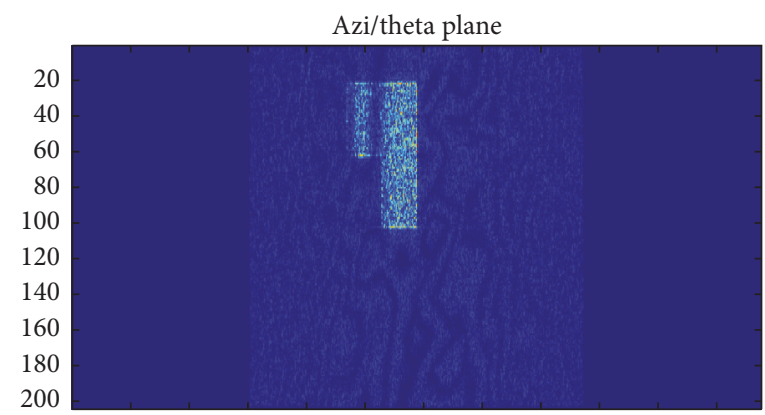

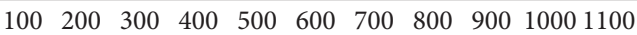

FIGURE 19: Azimuth-angle plane.

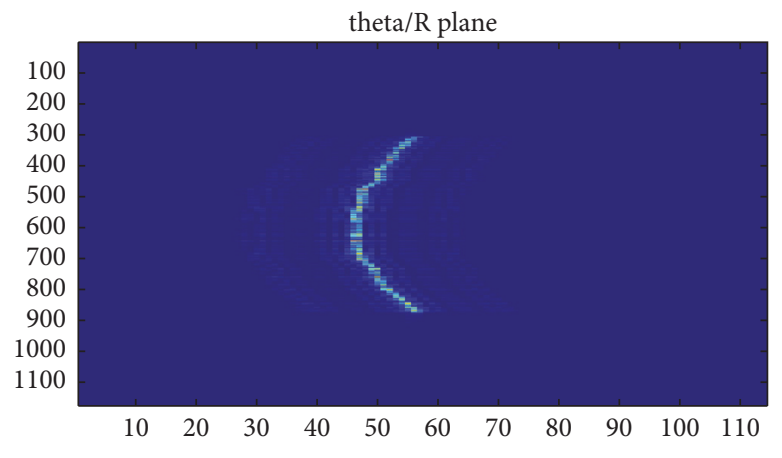

Figure 20: Angle-range plane.

The equivalent virtual array obtained by EPC principle is shorter than the actual physical array by $M-1$ unit array element distance, where $M$ is the number of transmitting antennas. So the transmitting array is

$\{0,2,4,6,8,10,12,14,16,18,20,22,24,26,28,1200,1202$,

$1204,1206,1208,1210,1212,1214,1216,1218,1220$,

$1222,1224,1226,1228\}$.
The receiving array is

$\{29,59,89,119,149,179,209,239,269,299,329,359,389$,

$419,449,479,509,539,569,599,629,659,689,719$,

$749,779,809,839,869,899,929,959,989,1019,1049$,

$1079,1109,1139,1169,1199\}$.

The imaging results corresponding to this proposed method are shown in Figures 24-29. 


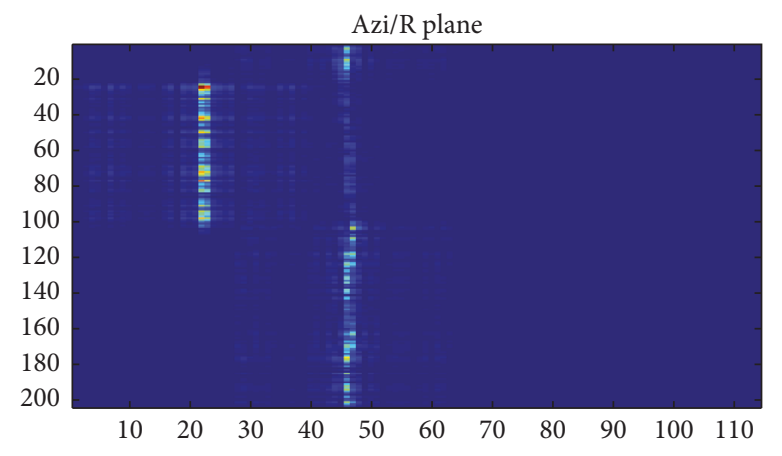

FIgURE 21: Azimuth-range plane.

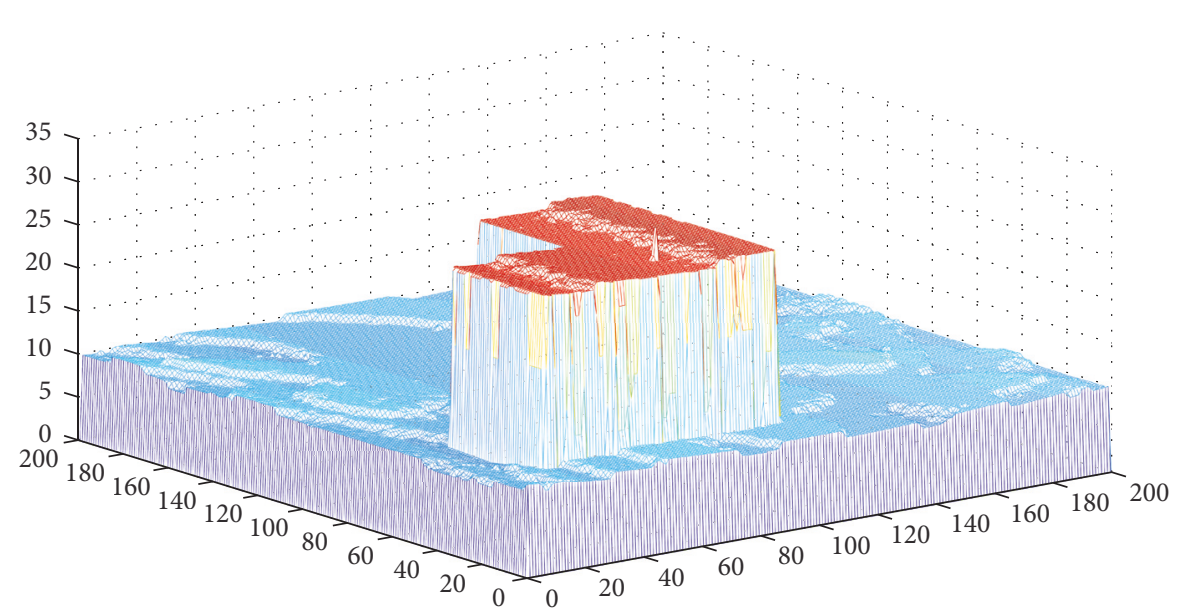

FIGURE 22: Imaging results.

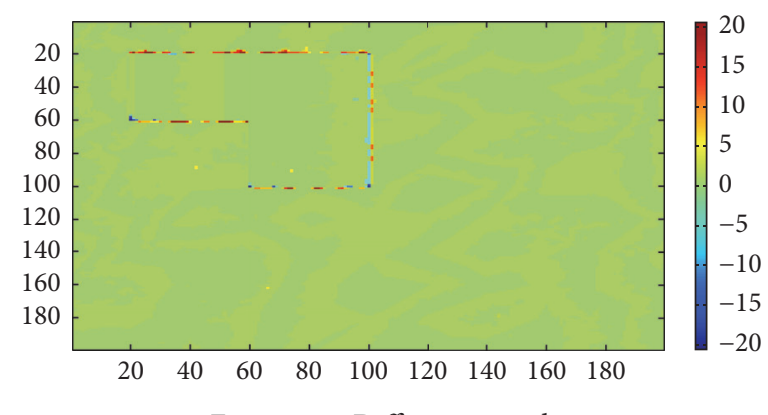

Figure 23: Difference results.

From the visual point, the imaging results using above two principles are very similar to those using the method in this paper. And these three methods can well restore the original appearance of the building. In order to quantitatively compare the imaging results of all three methods, Table 5 summarizes the mean and standard deviation of height reconstruction error eliminating the influence of shadow areas and the probability of height reconstruction error within half a resolution of the whole scene and the individual building area. The smaller the mean and standard deviation are and the greater the probability of the error within half a resolution is, the more accurate the elevation will be after imaging. In this paper, the theoretical resolution in the range direction is $1 \mathrm{~m}$ calculated by $\rho_{z}=c / 2 B$ [1] (p. 30). From the statistical values in Table 5 , the results indicators of EPC principle are higher than spatial convolution principle and this method, which are very limited. And the latter two are equivalent in all indicators. The main reason is that this method is evolved based on spatial convolution principle. When the equivalent uniform array is obtained using the two methods, the different transmitting and receiving array elements correspond to their specific equivalent position, resulting in the compensation phase of each target point being more complicated and more approximate than EPC principle. However, from both the visual point and the statistical values, this method is still able to get good imaging 


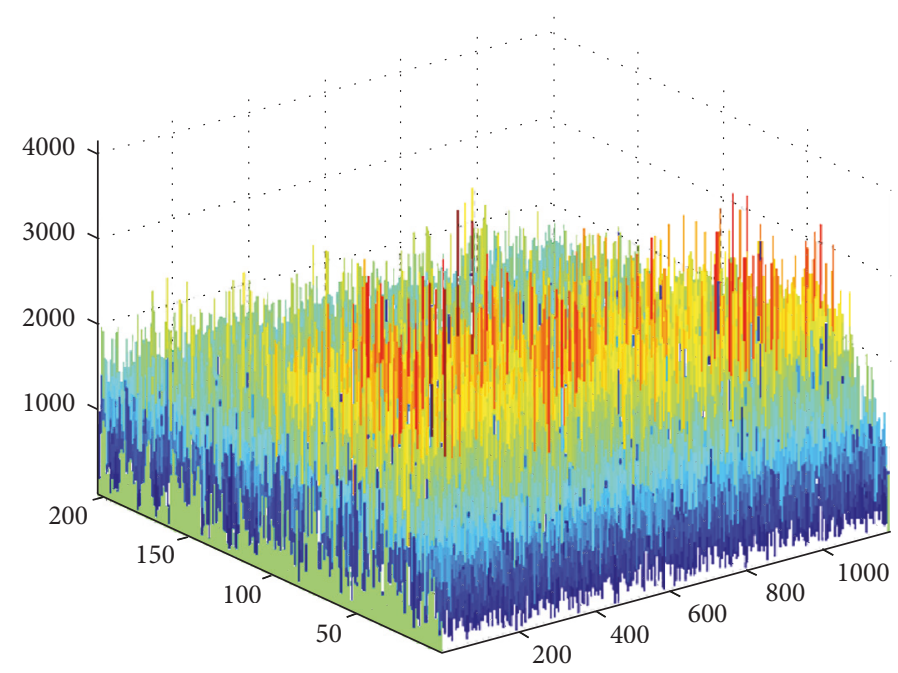

FIGURE 24: Original echo.

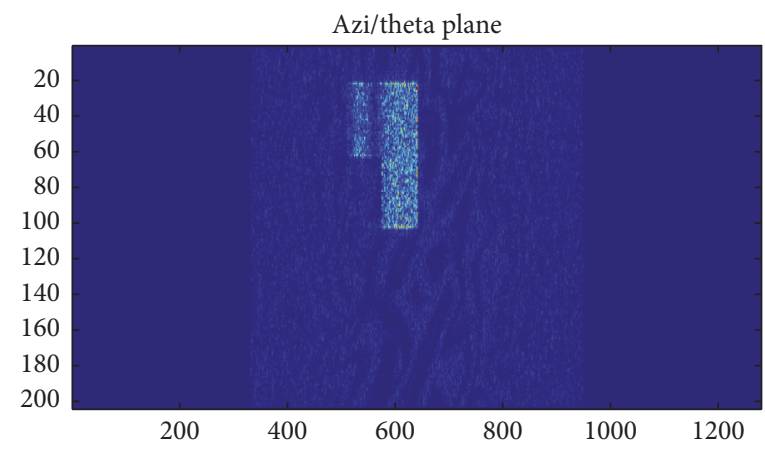

FIGURE 25: Azimuth-angle plane.

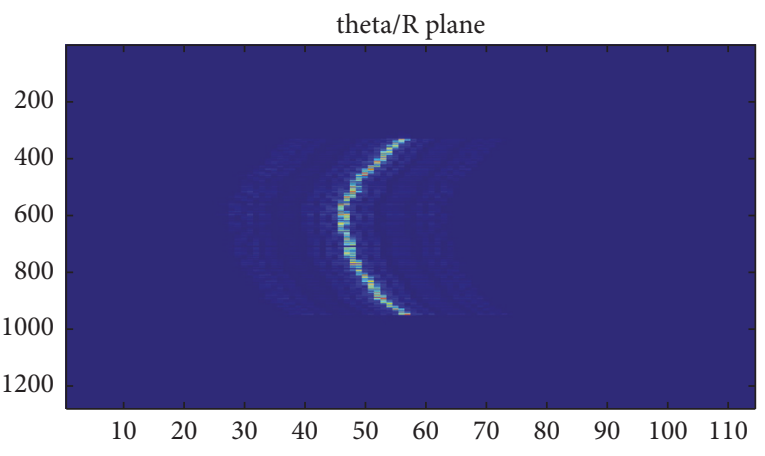

Figure 26: Angle-range plane.

results, which proves that the array design is reasonable and effective. This proposed method can provide another effective strategy for MIMO array design of array SAR.

\section{Conclusions}

The resolution of the cross-track for downward-looking array SAR depends on the array antenna length. The longer the

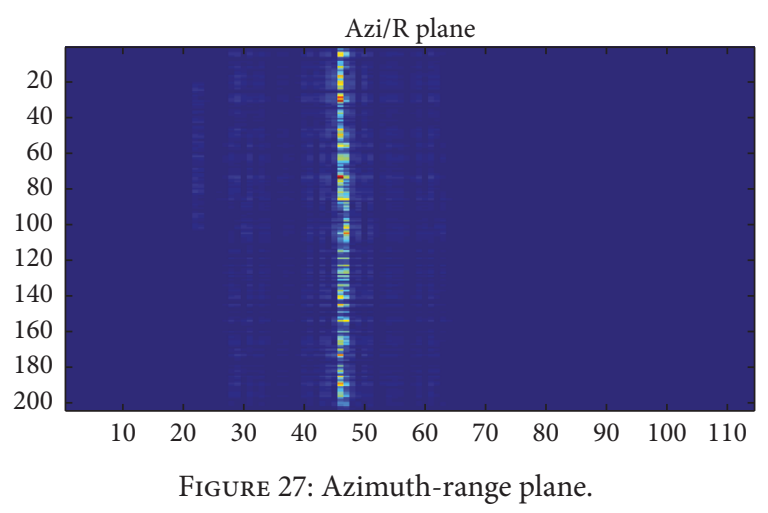

length of the array is, the higher the resolution of this dimension will be. Then, as the number of required array elements increases more, the design cost and the implementation complexity of the linear array antenna will double. In this paper, MIMO antenna polynomial weighted average design method of downward-looking array SAR is proposed by combining MIMO technology with array SAR. Furthermore, wave band, resolution, elevation precision, and working height concerned by surveying and mapping application are 
TABLE 5: Quantitative statistics indicators of 3D imaging results.

\begin{tabular}{lcccc}
\hline Imaging principle & $\begin{array}{c}\text { Mean of height } \\
\text { reconstruction } \\
\text { error/m }\end{array}$ & $\begin{array}{c}\text { Standard deviation of } \\
\text { height reconstruction } \\
\text { error/m }\end{array}$ & $\begin{array}{c}\text { Probability of height } \\
\text { reconstruction error within half a } \\
\text { resolution of the whole scene (\%) }\end{array}$ & $\begin{array}{c}\text { Probability of height reconstruction } \\
\text { error within half a } \\
\text { resolution of the individual building } \\
\text { area (\%) }\end{array}$ \\
\hline $\begin{array}{l}\text { EPC } \\
\begin{array}{l}\text { Spatial } \\
\text { convolution }\end{array}\end{array}$ & -0.0039 & 0.8485 & 99.27 & 94.15 \\
This method & -0.0147 & 0.8858 & 99.18 & 93.43 \\
\hline
\end{tabular}

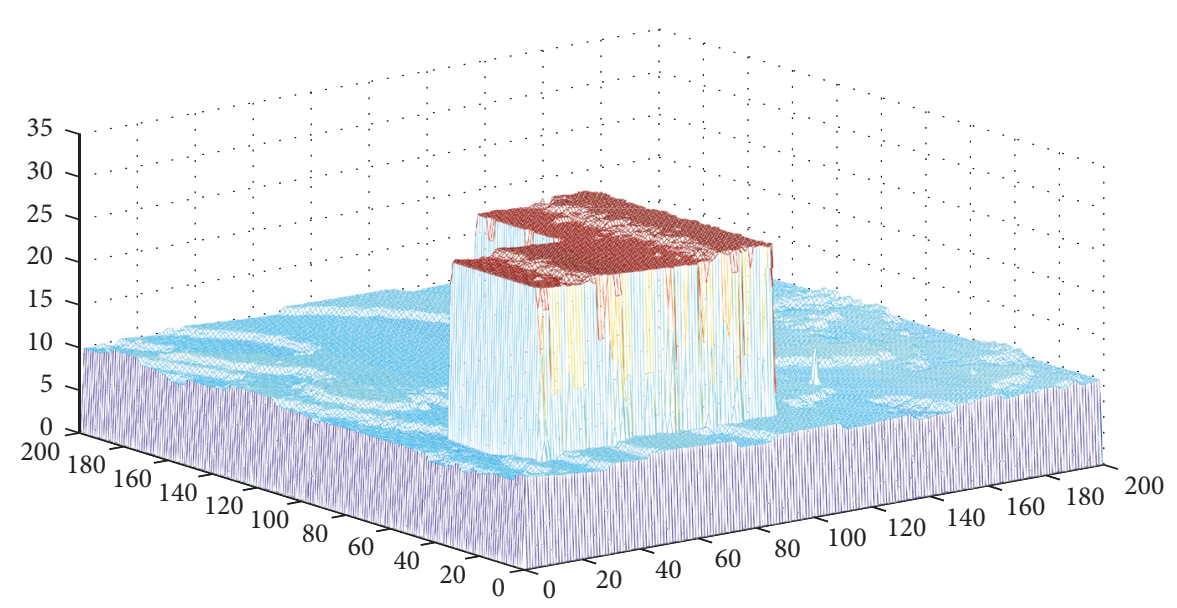

FIGURE 28: Imaging results.

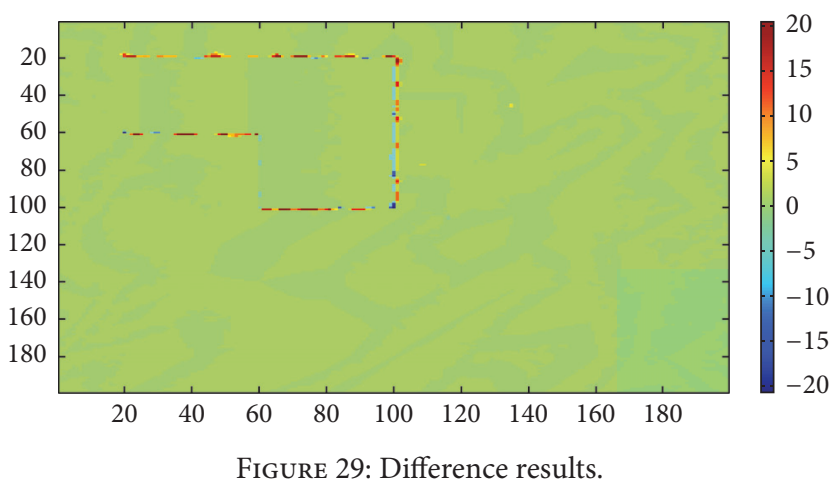

introduced, and the array number and position vectors of MIMO downward-looking array SAR of real mapping aerial platform are optimized. The simulation experiment proves the rationality and validity of the array design.

In order to promote the practical design of the array antenna, we need to further consider the influence of beam azimuth width and azimuth resolution on the size of subarray, the size of array, and the number of elements and further verify the elevation inversion accuracy, providing a solution to the ill-posed problem of complex array antenna design with MIMO mode.

\section{Conflicts of Interest}

The authors declare that there are no conflicts of interest regarding the publication of this paper.

\section{Acknowledgments}

This work was supported by the National Natural Science Foundation of China under Grant no. 41071296, no. 41474010, and no. 61401509. In addition, the authors would like to gratefully acknowledge Professor Liang-sheng Lou for his array SAR technical support.

\section{References}

[1] L. Du, Study on Model, Algorithm and Experiment for Downward-Looking Synthetic Aperture Radar Three-Dimensional Imaging Based on Linear Array Antennas, Institute of Electronics, Chinese Academy of Sciences, Beijing, China, 2010.

[2] Y. B. Wang, Research on Key Technologies of New ThreeDimensional Array SAR, University of Electronic Science and Technology of China, Chengdu, China, 2009. 
[3] J. H. G. Ender, "Along-track array processing for MIMO-SAR/ MTI," in Proceedings of the European Conference on Synthetic Aperture Radar (EUSAR '08), pp. 4032-4036, Friedrichshafen, Germany, 2008.

[4] L. B. White and P. S. Ray, "Signal design for MIMO diversity systems," in Proceedings of the Conference Record of the 38th Asilomar Conference on Signals, Systems and Computers, pp. 973-977, Pacific Grove, Calif, USA, November 2004.

[5] F. C. Robey, S. Coutts, D. Weikle, J. C. McHarg, and K. Cuomo, "MIMO radar theory and experimental results," in Proceedings of the Conference Record of the 38th Asilomar Conference on Signals, Systems and Computers, pp. 300-304, Lexinton, Ky, USA, November 2004.

[6] A. Bellettini and M. A. Pinto, "Theoretical accuracy of synthetic aperture sonar micronavigation using a displaced phase-center antenna," IEEE Journal of Oceanic Engineering, vol. 27, no. 4, pp. 780-789, 2002.

[7] M. Weiß and J. H. G. Ender, "A 3D imaging radar for small unmanned airplanes-ARTINO," in Proceedings of the 2nd European Radar Conference ( EURAD '05), pp. 209-212, IEEE, Paris, France, October 2005.

[8] J. Klare, "A new airborne radar for 3D imaging-simulation study of ARTINO," in Proceedings of the European Conference on Synthetic Aperture Radar (EUSAR '06), pp. 3842-3845, Dresden, Germany, 2006.

[9] J. Klare, D. Cerutti-Maori, A. Brenner, and J. Ender, "Image quality analysis of the vibrating sparse MIMO antenna array of the airborne 3D imaging radar ARTINO," in Proceedings of the IEEE International Geoscience and Remote Sensing Symposium (IGARSS '07), pp. 5310-5314, Barcelona, Spain, June 2007.

[10] M. Weiß, O. Peters, and J. Ender, "First flight trials with ARTINO," in Proceedings of the 7th European Conference on Synthetic Aperture Radar (EUSAR '08), pp. 1-4, Friedrichshafen, Germany, June 2008.

[11] M. Weiß and M. Gilles, "Initial ARTINO radar experiments," in Proceedings of the 8th European Conference on European Conference on Synthetic Aperture Radar (EUSAR '10), pp. 1-4, Aachen, Germany, 2010.

[12] Y. Su, Y. T. Zhu, W. X. Yu et al., "Design theory and algorithm of multichannel radar antenna arrays," Chinese Science: Information Science, vol. 40, pp. 1372-1383, 2010.

[13] S. K. Mitra, K. Mondal, M. K. Tchobanou, and G. J. Dolecek, "General polynomial factorization-based design of sparse periodic linear arrays," IEEE Transactions on Ultrasonics, Ferroelectrics, and Frequency Control, vol. 57, no. 9, pp. 1952-1966, 2010.

[14] A.-L. Chen, D.-W. Wang, X.-Y. Ma, and Y. Su, "Array design and imaging method for wide-band MIMO radar," Systems Engineering and Electronics, vol. 32, no. 12, pp. 2541-2546, 2010.

[15] J. Zhang, L.-R. Zhang, and N. Liu, "Array optimization algorithm for MIMO radar with a maximum physical sensors efficiency," Journal of Xidian University, vol. 37, no. 1, pp. 86-90, 2010.

[16] Q. Wang, Study on Approaches of MIMO Radar Array Design,, National University of Defense Technology, Changsha, China, 2011.

[17] Y. Ye, G. Y. Liu, and Z. Meng, "Airborne downward-looking 3D-SAR systems and its imaging using sparse array antennas," Journal of CAEIT, vol. 6, pp. 96-100, 2011.

[18] X.-M. Peng, Y.-P. Wang, W.-X. Tan, W. Hong, and Y.-R. Wu, "Airborne downward-looking MIMO 3D-SAR imaging algorithm based on cross-track thinned array," Journal of Electronics and Information Technology, vol. 34, no. 4, pp. 943-949, 2012.
[19] Y. T. Li, Y. G. Chen, Y. K. Deng, and C. B. Yin, "A study on airborne MIMO-SAR linear array antenna configuration," Modern Radar, vol. 34, pp. 4-7, 2012.

[20] W.-Q. Wang, H. Shao, and J. Cai, "MIMO antenna array design with polynomial factorization," International Journal of Antennas and Propagation, vol. 2013, Article ID 358413, 9 pages, 2013.

[21] Z.-B. Wu, Y.-T. Zhu, Y. Su, Y. Li, and X.-J. Song, "MIMO array design for airborne linear array 3D SAR imaging," Journal of Electronics and Information Technology, vol. 35, no. 11, pp. 26722677, 2013.

[22] C. Y. Yu, Y. N. Xie, H. L. Zhao et al., "Airborne downward-looking MIMO 3D-SAR imaging properties based on the new array configuration," Electronic Measurement Technology, vol. 37, pp. 64-69, 2014.

[23] D. J. Li, Y. N. Hou, X. M. Teng et al., Radar Technology and Application of Sparse Array Antenna, Science Press, Beijing, China, 1st edition, 2014.

[24] D. W. Bliss and K. W. Forsythe, "Multiple-input multiple-output (MIMO) radar and imaging: degrees of freedom and resolution," in Proceedings of the 37th Asilomar Conference on Signals, Systems and Computers, pp. 54-59, Pacific Grove, Calif, USA, November 2003.

[25] National Administration of Surveying and Mapping, " $\mathrm{CH} / \mathrm{Z}$, basic geographic information three-dimensional model product specification (draft)," Standard, Surveying and Mapping Press, Beijing, China, 2012.

[26] National Administration of Surveying and Mapping, "Lowaltitude digital aerial photography specification," Standard CH/Z 3005-2010, Surveying and Mapping Press, Beijing, China, 2010. 


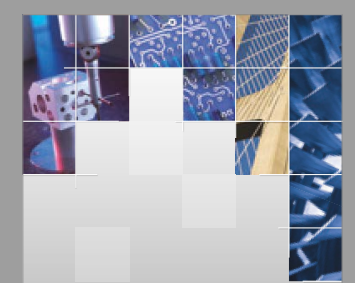

\section{Enfincering}
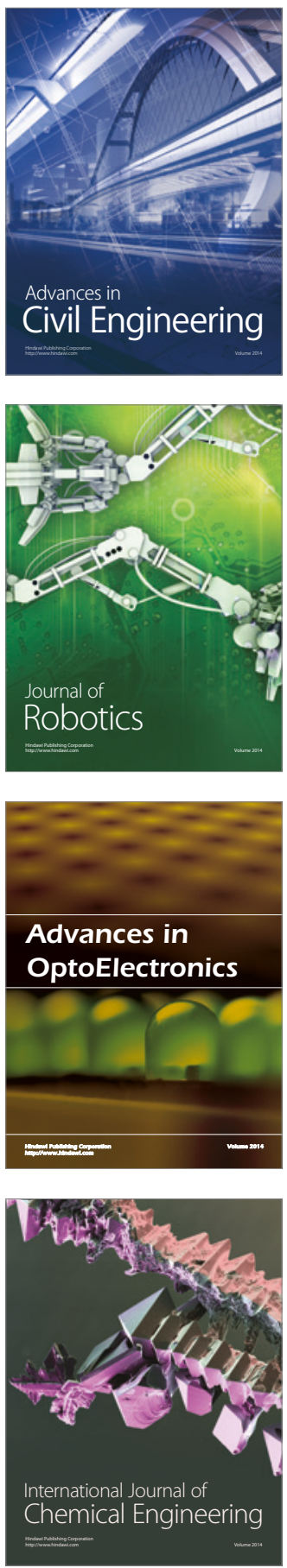

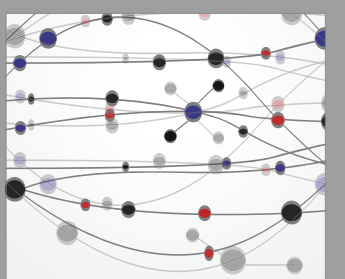

The Scientific World Journal

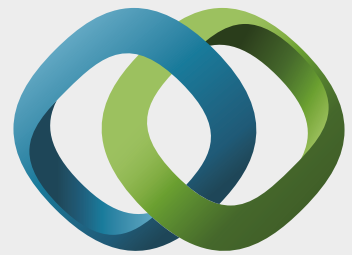

\section{Hindawi}

Submit your manuscripts at

https://www.hindawi.com
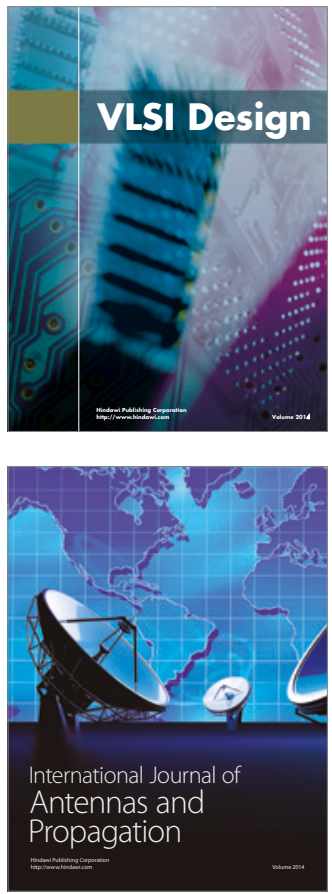

\section{Rotating}

Machinery
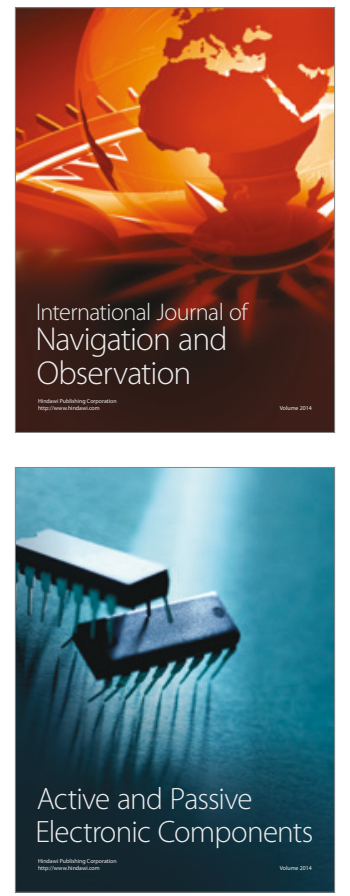
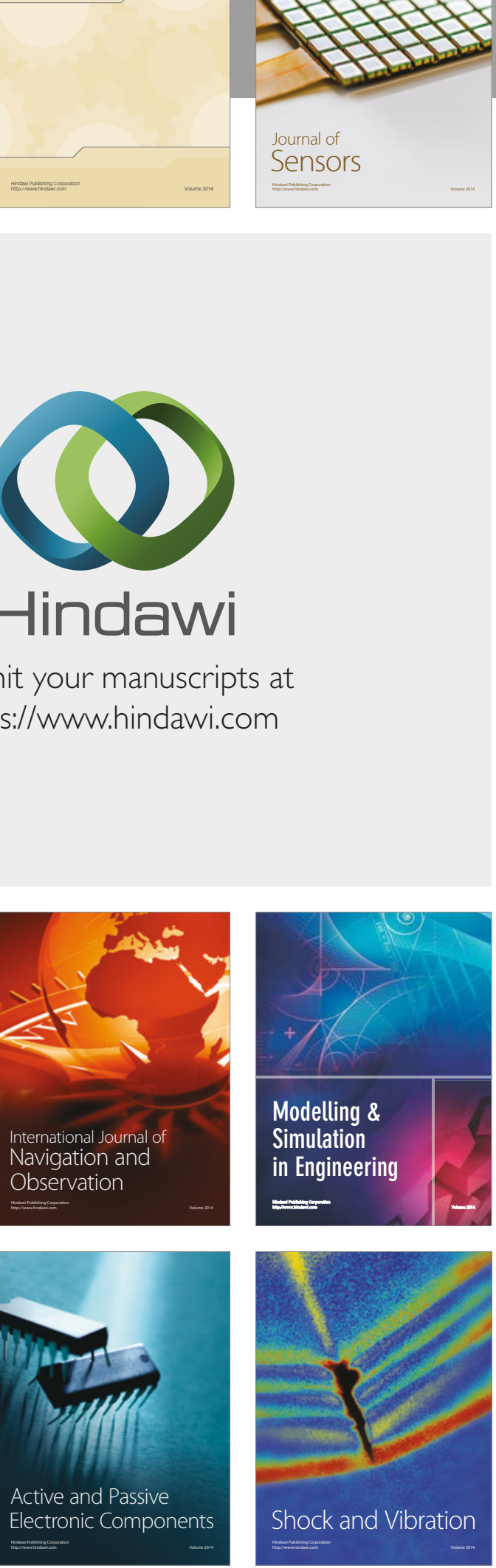
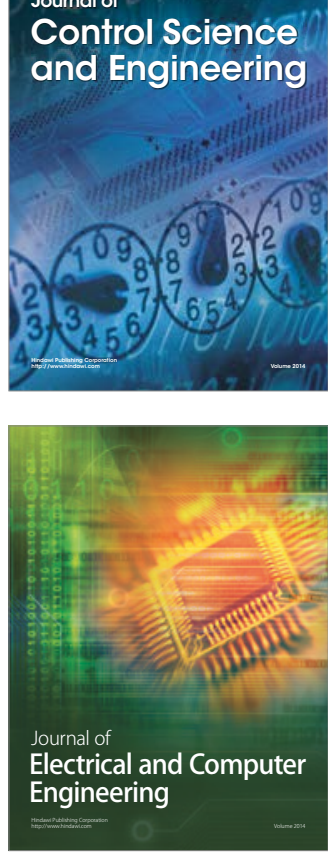

Distributed

Journal of

Control Science

and Engineering
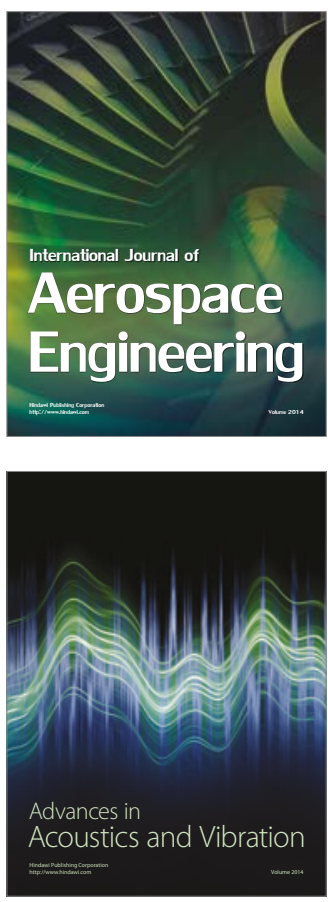

Sensor Networks 